\title{
LA TRANSICIÓN AL MUNDO LABORAL DE LOS JÓVENES CON DEFICIENCIAS AUDITIVAS
}

\author{
ENTRY INTO WORKING LIFE OF YOUTH WITH \\ HEARING DIFFICULTIES
}

\author{
M. Miguel* y M. Pereira** \\ Universidad de Oviedo ${ }^{1}$
}

\section{RESUMEN}

Este artículo forma parte de una investigación más amplia sobre los procesos de inserción laboral de los jóvenes con deficiencias auditivas. En él presentamos las principales conclusiones del análisis documental que efectuamos sobre las investigaciones y estudios realizados sobre personas con problemas auditivos y sus dificultades de inserción sociolaboral. En primer lugar, clarificamos la terminología que se utiliza en este campo, abordamos el tema de la clasificación de las deficiencias auditivas y nos referimos también a la incidencia de este problema. Posteriormente nos centramos en la investigación sobre personas con problemas auditivos: 1) las dificultades asociadas a las deficiencias auditiva; 2) la relevancia de la formación de estas personas; 3) la necesidad de apoyo y orientación para el trabajo; 4) las principales opciones vinculadas a la comunicación y a la integración; 5) la sensibilización de la población ante las dificultades auditivas y 6) la significatividad de las nuevas tecnologías para este colectivo. Para terminar, detallamos las recomendaciones que deben tenerse en cuenta al formar o trabajar con personas que tienen problemas auditivos.

Palabras clave: orientación y formación de jóvenes con dificultades auditivas, transición al mundo laboral, investigación sobre personas con deficiencias auditivas

1. Investigación realizada mediante Contrato de Investigación entre la Dirección Provincial del MEC en Asturias y la Universidad de Oviedo por los autores de este artículo, con la colaboración de personal docente de la Fundación Vinjoy: Alicia Portilla, Adolfo Rivas y María Jesús Valdivieso.

* Catedrático de Métodos de Investigación y Diagnóstico en Educación de la Universidad de Oviedo. Ha realizado numerosos trabajos de investigación evaluativa, entre los que destacan dos proyectos que dirige actualmente con objeto de evaluar los Centros y Entidades que desarrollan los Planes FIP en Asturias y la institución educativa que tradicionalmente acoge en Asturias a los huérfanos de la minería (FUNDOMA).

** Profesora Titular de Orientación Profesional y Ocupacional de la Universidad De Oviedo. La mayor parte de sus trabajos se vinculan al campo de la educación y la orientación para el trabajo. Forma parte del equipo que desarrolla los proyectos de evaluación dirigidos por el Profesor De Miguel.

Dirección de contacto: Facultad de Ciencias de la Educación, C/ Aniceto Sela s/n, 33005 Oviedo. Direcciones electrónicas: mario@pinon.ccu.uniovi.es y pereira@correo.uniovi.es 


\section{ABSTRACT}

This article is part of a broader research about the processes of insertion in the world of work of the youth with hearing difficulties. We present the main conclusions of the documentary analysis that we made about the investigations and studies carried out with people with auditory problems and their difficulties of social and labour integration. In first place, we clarify the terminology that is used in this field, we approach the issue of the classification of the auditory deficiencies and we also refer to the incidence of this problem. Next we analyse the investigation about people with hearing difficulties: 1) the difficulties associated to the auditory problems; 2) the relevance of the formation of these people; 3) the need of support and career guidance; 4) the main options linked to the communication and the integration; 5) the sensitising of the population about the hearing difficulties, and 6) the importance of the new technologies for this community. To finish, we detail the recommendations that should be kept in mind when we are forming or working with people with auditory problems.
\end{abstract}

Key words: guidance and training of youth with hearing difficulties, transition to the labor world, research about people with auditory problems.

\title{
Introducción
}

En este trabajo presentamos una parte significativa de los resultados de un amplio estudio de evaluación de las necesidades de inserción laboral de los jóvenes con problemas auditivos. En concreto, nos centramos en el primer objetivo de este estudio, en el que planteamos la conveniencia de "efectuar un análisis documental sobre la investigación y estudios realizados con personas con deficiencias auditivas, con el fin de recabar información actualizada sobre las principales dificultades que experimentan en sus procesos de inserción laboral y sobre los aspectos más relevantes y decisivos para dichos procesos».

Para poder efectuar este análisis documental realizamos una amplia revisión bibliográfica, identificando, localizando y analizando documentación de distinto tipo: libros y artículos de revistas, información procedente de direcciones electrónicas especializadas en discapacidad o deficiencias auditivas y de otras páginas Web de interés, e informes provenientes de bases de datos sobre educación.

En este artículo abordamos los aspectos más relevantes de dicho análisis. Partimos de la necesidad de clarificar la terminología que se utiliza en este campo, la categorización de las dificultades auditivas y la incidencia de este problema en la población. Posteriormente nos centramos en el análisis de las investigaciones y estudios realizados en relación con el tema, abordando de manera específica:

a) las dificultades asociadas a las deficiencias auditivas;

b) la importancia de la formación;

c) la necesidad de apoyo y orientación para el trabajo;

d) las principales opciones relativas a la comunicación y a la integración;

e) la urgencia de sensibilizar a la población ante las dificultades auditivas; y

f) la significatividad de las aportaciones de las nuevas tecnologías para este colectivo.

Por último, destacamos las implicaciones prácticas de este análisis, subrayando las recomendaciones que deben tenerse en cuenta a la hora de formar o trabajar con personas que presentan deficiencias auditivas. 


\section{Clarificación terminológica}

Una somera aproximación al campo pone de manifiesto la confusión terminológica y la ausencia de un consenso generalizado sobre los conceptos y sobre la forma en la que nos referimos a las personas con diversos tipos de deficiencias y, particularmente, a las que tienen dificultades auditivas. En nuestro contexto se utilizan una gran pluralidad de términos y existe cierto confusionismo como fácilmente se puede observar en:

1. las publicaciones y en la literatura especializada sobre el tema (libros, artículos de revistas científicas, folletos informativos y divulgativos, prensa...);

2. las nomenclaturas adoptadas por la Administración y por las distintas instituciones y asociaciones en muchos de sus servicios y programas dirigidos a estos colectivos; $\mathrm{y}$

3. el lenguaje habitual utilizado en los procesos de comunicación oral (entre expertos, profesionales, políticos, familiares, amigos y el cotidiano empleado a un nivel más general en la sociedad).

Tampoco fuera de España este problema está resuelto y ni siquiera los representantes de los grandes organismos internacionales implicados han podido llegar a un consenso sobre el sistema de clasificación propuesto por la Organización Mundial de la Salud y sobre las definiciones que resultan más adecuadas (Comisión Europea, 1996).

La relatividad histórica y cultural de las categorías que se han utilizado como referente de las personas con distintos tipos de deficiencias ha sido bien descrita en publicaciones recientes (Aguado, 1995; Pereda y otros, 1998), que reflejan la evolución conceptual de este campo en diversos momentos y contextos históricos. Uno de los cambios más significativos que a este respecto merece la pena destacar, es el que se produjo en los años 60 cuando -frente a los planteamientos anteriores de etiquetado, institucionalización y protección especial-, se comenzó a hacer hincapié en los condicionamientos psicosociales y socioambientales de las deficiencias, en la necesidad de prevención, en la importancia de la desinstitucionalización de estas personas y en la conveniencia de conseguir su verdadera inserción comunitaria.

En España los cambios que se estaban produciendo fuera de nuestras fronteras tuvieran lugar un poco más tarde. El referente más significativo, al que siempre aludimos, lo constituye la publicación en 1982 de la Ley de Integración Social de los Minusválidos (LISMI) que intenta garantizar para estos colectivos los derechos universales que con la Constitución Española de 1978 se reconocieron para todos los españoles. Con esta Ley se trata de establecer un marco legal y una política activa de integración social de los "disminuidos» en sus capacidades físicas, psíquicas y sensoriales (con una política de rehabilitación y normalización sociolaboral) y continuar asistiendo y tutelando a los "disminuidos profundos» (con una política de protección especial -pensiones y prestaciones- y etiquetado).

El posterior Plan de Acción Integral para las Personas con Discapacidad (INSERSO, 1996), que enfatiza la necesidad de desarrollar políticas más activas de integración sociolaboral, también refleja los cambios que se están produciendo en la terminología que utilizamos en nuestro contexto: frente a los términos «minusválido» y «disminuido» empleados en la LISMI se habla, como en muchos otros documentos y publicaciones, de «personas con discapacidad». Para comprender las connotaciones de estos conceptos, es necesario referirnos a la Clasificación Internacional propuesta por la Organización Mundial de la Sa- 
lud (OMS) en 1980, que fue elaborada para ser aplicada en el área de la salud y en el tratamiento de las enfermedades (Organización Mundial de la Salud, 1994). La versión en castellano de esta clasificación fue promovida por el INSERSO en 1983, utilizando los términos: "deficiencias», "discapacidades» y «minusvalías». La LISMI no se ajustó completamente a las categorías de la clasificación de la $O M S$ y consideramos que la traducción a nuestro idioma de esta clasificación -que ha ejercido una influencia notable en los usos lingüísticos en este campo hasta la fecha-, tampoco es la más adecuada: impairment (deficiencia), disability (discapacidad) y handicap (minusvalía).

Siguiendo esta argumentación, es necesario comprender cómo estos tres conceptos de la clasificación de la $O M S$ remiten a tres planos diferentes en la valoración de los efectos negativos de la enfermedad que pueden incidir sobre una persona. Pereda y otros (1998) se refieren a ellos de este modo:

- Deficiencia: cualquier pérdida o anormalidad de una estructura o función anatómica, fisiológica o psicológica. Las deficiencias son resultado del desarrollo de enfermedades, traumatismos o trastornos de cualquier tipo (incluso genéticos y perinatales), pero no siempre tales situaciones producen deficiencias (sólo una parte de las enfermedades se traducen en deficiencias persistentes). Representan trastornos a nivel de órgano.

- Discapacidad: cualquier restricción o ausencia de capacidad funcional para realizar actividades cotidianas en la forma o dentro del margen que se considera normal para un ser humano. Las discapacidades son siempre consecuencia de una deficiencia, pero éstas a veces no producen discapacidad (hay más deficiencias que discapacidades). Representan trastornos funcionales a nivel de la persona.

- Minusvalía: desventaja social que experimenta una persona como consecuencia de las deficiencias y discapacidades, lo que limita o impide el desempeño de un rol que es normal en función de su edad, sexo y otros factores culturales. Las minusvalías implican como condición necesaria, pero no suficiente, la existencia previa de deficiencias y discapacidades. Además es preciso que existan circunstancias y barreras específicas que coloquen a esos sujetos en una posición de desventaja con respecto a los grupos sociales de su entorno (dos personas con idéntica discapacidad pueden tener o no una minusvalía). Las minusvalías representan trastornos o problemas a nivel de la inserción en la sociedad.

De esta forma, la propuesta de la $O M S$ contribuye a superar los enfoques médicos tradicionales en la medida en que contempla y destaca la influencia decisiva de los contextos sociales en la inserción sociolaboral de las personas con discapacidad. Pero se le critica el olvidar la posible etiología social de los dos primeros conceptos y la ambigüedad del componente social atribuida al tercero (Pereda y otros, 1998).

A estas consideraciones hay que añadir otras que nos parecen muy importantes en relación con el término elegido para referirse a «handicap», en castellano: "minusvalía». Sin perder de vista la base del significado del sistema clasificatorio de la $O M S$-que atribuye a los tres términos un componente orgánico, funcional y social respectivamente-, el planteamiento de McBain y Phillips-Jones (1985) contribuye a la clarificación terminológica que pretendemos plantear. Estas autoras destacan la importancia de percibir a estos colectivos con los que trabajamos como "persons with disabilities», en lugar de "handicapped-persons». El primer término subraya, ante todo y fundamentalmente, su condición de personas 
y sólo con posterioridad sus dificultades o discapacidades. La segunda expresión, en cambio, enfatiza prioritariamente sobre cualquier otro aspecto la condición problemática del sujeto. En este trabajo también se destaca que una dificultad funcional (disability), sólo se convierte en un handicap, impedimento o desventaja, cuando verdaderamente supone un obstáculo real para la consecución de los objetivos de la persona².

Consideramos esta línea de argumentación como muy importante ya que contribuye a disminuir la utilización de la palabra «minusvalía». Aunque este término se emplea frecuentemente en nuestro contexto, sus connotaciones son extremadamente negativas y estigmatizadoras. Su uso ha sido, y continúa siendo, tan habitual y está tan «institucionalizado», que no parece que quienes lo emplean lo hayan analizado de forma suficientemente crítica. Decir que alguien es "minusválido" supone establecer un juicio de valor en el que la palabra que utilizamos indica que, por el motivo que sea, esa persona "vale-menos» que otra que no comparte su condición.

En nuestro país la utilización de esta expresión está socialmente muy extendida y también forma parte del vocabulario habitual que se emplea en los servicios y programas del Ministerio de Trabajo y Asuntos Sociales y de las Consejerías correspondientes a nivel autonómico. De hecho se suele emplear en la mayoría de las actuaciones de la Administración "dirigidas a discapacitados»: los Centros de atención para personas afectadas de minusvalía, los Centros de recuperación de minusválidos físicos (CRMF), los Centros de atención de minusválidos fisicos (CAMF), los Centros de atención a minusválidos psíquicos (CAMP), las Prestaciones de integración social de los minusválidos (LISMI) o Ayudas y subvenciones para la realización de programas para personas con discapacidad. (Ministerio de Trabajo y Asuntos Sociales, 2000). Como ya hemos indicado, la palabra «minusvalía» es un término poco afortunado, que contribuye a segregar en lugar de a integrar y que indica una actitud social contraria al espíritu normalizador consensuado en este campo -al menos en el plano teórico- y también contraria al respeto a la diversidad al que tanto aludimos en los últimos años. Sea cual sea la característica y/o dificultad que haga a alguien diferente de los demás, e independientemente de su grado y de la persistencia, magnitud o gravedad de sus efectos, nunca debería ser utilizada para hacerla sentirse más o menos válida, mejor o peor que otras personas, sino sólo, en todo caso, distinta o diferente.

Por otro lado, tampoco estamos de acuerdo con la forma en la que se generan etiquetas a partir de los conceptos que abordamos: "disminuido", "deficiente», "discapacitado", "minusválido», "sordo", "ciego», "retrasado mental» ... El uso que se hace de estos términos para aludir a las personas con dificultades sensoriales, físicas y psíquicas, enfatiza en primer lugar y sobre todo, la característica que les hace diferentes a los demás: la parte (la característica diferencial) se utiliza para definir al todo (a la persona) y consigue desdibujarlo por su carácter claramente parcial, centrado exclusivamente en el problema. Por eso resulta más adecuado hablar de «personas con dificultades sensoriales, físicas o psíquicas» o de "personas con deficiencias o discapacidades»: se enfatiza principalmente la idea de persona, como un conjunto holístico con múltiples características, con limitaciones, pero también con potencialidades, y sólo en segundo lugar se señala

2. Esta es la dirección hacia la que están evolucionando las tendencias en el campo de las discapacidades. Gregory (1992) desde Inglaterra realizó un estudio en el que define las categorías de la sordera no en términos del grado de la pérdida auditiva sino de sus consecuencias para la persona con deficiencia auditiva. 
su particularidad o condición diferencial, efectivamente importante, pero que forma parte de dicho conjunto ${ }^{3}$. Aunque se están produciendo cambios en el uso de la terminología, de momento, coexisten con usos tradicionales menos adecuados. El proceso es lento, pero no cabe duda que los cambios se siguen afianzando como se puede observar en las publicaciones, proyectos desarrollados y encuentros de profesionales durante los últimos. Por ello, creemos que términos como sordos, minusválidos, deficientes auditivos, discapacitados auditivos son inadecuados y que es más conveniente hablar de personas con dificultades auditivas, personas con deficiencias auditivas, personas con discapacidad auditiva o de personas sordas.

\section{Clasificación de las deficiencias auditivas}

Esta heterogeneidad y diversidad en el uso de los conceptos se constata igualmente cuando se hace referencia a las personas sordas, tal como se puede observar en las clasificaciones que se realizan al respecto. Así, en la versión de la Encuesta sobre Deficiencias, Discapacidades y Minusvalías que hizo en 1986 el Instituto Nacional de Estadística de la clasificación de la OMS de 1980 (Instituto Nacional de Estadística, 1987), los problemas auditivos aparecen categorizados tal como lo recoge la Tabla 1.

Pereda y otros (1998), partiendo de esta categorización y de otros sistemas de clasificación utilizados en nuestro país, elaboraron un Registro homologado de la discapacidad y de su tratamiento y de los efectos sociales subsiguientes, con el objeto de disponer de información precisa y objetiva sobre la discapacidad. En este registro se definen los problemas auditivos (deficiencias y discapacidades) como se refleja en la Tabla 2.

En otro trabajo, recientemente publicado por Fondo-Formación (Flórez García y otros, 1998), al abordar la clasificación de las personas con deficiencias auditivas - a cuyos deficits denominan hipoacusias- elaboran una clasificación en función de tres criterios:

a) la localización de la lesión;

b) la cantidad de pérdida auditiva; $y$

c) el momento de adquisición de la deficiencia auditiva (Tabla 3).

\section{Incidencia del problema}

Concretar de forma exacta cuántas personas de la población están afectadas por los distintos tipos de dificultades sensoriales, físicas o psíquicas siempre ha sido un problema difícil de resolver y continúa siéndolo en la actualidad. La mencionada Encuesta sobre Defi-

3. El mismo argumento se aplica a otros términos, como el de «superdotado» que, de la misma forma, desdibuja la idea global de la persona (el todo) a través de una de sus características (la parte). La diferencia, que también ilustra nuestra argumentación, es que las connotaciones sociales atribuidas a la idea de «superdotación» se suelen percibir de forma positiva y no negativa como las asociadas a los términos a los que nos estamos refiriendo.

Recordemos por ejemplo, que en la última Guía Laboral y de Asuntos Sociales del MTAS se habla al mismo tiempo de minusválidos, minusválidos físicos, minusválidos psíquicos, discapacitados, personas afectadas de minusvalia y de personas con minusvalía. 
TABLA 1: Esquema extraído de la clasificación de la Encuesta de Deficiencias, Discapacidades y Minusvalías del INE, detallando únicamente las referencias a los problemas auditivos.

\begin{tabular}{|c|c|c|}
\hline DEFICIENCIAS & DISCAPACIDADES & MINUSVALÍAS \\
\hline $\begin{array}{l}\text { 1. PSÍQUICAS } \\
\text { 2. SENSORIALES } \\
\text { - De la audición } \\
\text { Sordera total } \\
\text { Sordera de un } \\
\text { Oído } \\
\text { Mala audición } \\
\text { - De la vista } \\
\text { - Del lenguaje } \\
\text { 3. FISSICAS } \\
\text { 4. MIXTAS } \\
\text { 5. NINGUNA EN } \\
\text { ESPECIAL } \\
\text { 6. NO CONSTA }\end{array}$ & $\begin{array}{l}\text { 1. VER } \\
\text { 2. OÍR } \\
\text { - Sordo total } \\
\text { - Sordo de un oído } \\
\text { - Mala audición } \\
\text { 3. HABLAR } \\
\text { 4. OTRAS COMUNICACIÓN } \\
\text { 5. CUIDADO PERSONAL } \\
\text { 6. ANDAR } \\
\text { 7. SUBIR ESCALERAS } \\
\text { 8. CORRER } \\
\text { 9. SALIR DE CASA } \\
\text { 10. ACTIVIDADES DE LA VIDA } \\
\text { COTIDIANA } \\
\text { 11. DEPENDENCIA RESISTENCIA } \\
\text { 12. AMBIENTAL } \\
\text { 13. CONDUCTA CON UNO MISMO } \\
\text { 14. CONDUCTA CON LOS DEMÁS } \\
\text { 15. POSIBLES DISCAPIDADES } \\
\text { FUTURAS }\end{array}$ & $\begin{array}{l}\text { 1. DE ORIENTACIÓN } \\
\text { 2. INDEPENDENCIA } \\
\text { FÍSICA } \\
\text { 3. DE MOVILIDAD } \\
\text { 4. DE OCUPACIÓN } \\
\text { 5. DE INTEGRACIÓN } \\
\text { SOCIAL } \\
\text { 6. DE INSUFICIENCIA } \\
\text { ECONÓMICA } \\
\text { 7. NINGUNA } \\
\text { 8. NO CONSTA }\end{array}$ \\
\hline
\end{tabular}

TABLA 2: Definiciones de deficiencias y discapacidades auditivas extraídas del «Registro homologado de la discapacidad y de su tratamiento y de los efectos sociales subsiguientes» de Pereda y otros (1998).

\section{DEFICIENCIAS SENSORIALES}

- SORDERA TOTAL: incluye a las personas cuya deficiencia en ambos oídos es tan severa que no pueden beneficiarse de ninguna ampliación.

- SORDERA DE UN OÍDO: Incluye a las personas con la misma deficiencia que el grupo anterior pero referida a un solo oído.

- MALA AUDICIÓN: incluye a las personas que sin el uso de algún aparato no pueden mantener una conversación breve en tono normal.

\section{DISCAPACIDAD: OIR}

- SORDO TOTAL: incluye a las personas que padecen sordera total en ambos oídos.

- SORDO DE UN OÍDO: incluye a las personas que padecen sordera total en un solo oído.

- MALA AUDICIÓN: incluye a las personas que sin ayuda de aparato no pueden seguir una conversación en tono normal o tienen una grave dificultad para hacerlo. Las personas sordas de un solo oído y con mala audición en el otro están incluidas en el apartado anterior y en este, es decir, padecen dos discapacidades para oír. Los sordos totales no se incluyen en esta discapacidad 


\section{TABLA 3: Esquema elaborado a partir de la clasificación de las deficiencias auditivas} o hipoacusias de Flórez García y otros (1998).

\section{LOCALIZACIÓN (dónde está la lesión)}

1. HIPOACUSIA CONDUCTIVA O DE TRANSMISIÓN: afecta al oído externo y/o oído medio; suele ser un problema de no muy difícil solución; puede estar causada por factores congénitos, es decir, presentes en el momento del nacimiento (huesecillos anormales), por factores traumáticos (desgarros), inflamatorios (otitis), cerumen, etc.

2. HIPOACUSIA NEUROSENSORIAL O DE PERCEPCIÓN: se debe a una lesión a nivel de oído interno, o bien debida a problemas que afectan a la cóclea, al nervio auditivo o a las zonas auditivas del cerebro; el daño es más grave que en las anteriores; puede ser genética (hereditaria) o adquirida (por efecto de antibióticos, por traumatismos, procesos inflamatorios, etc.).

3. HIPOACUSIA MIXTA: afecta tanto al oído externo y/o medio como al oído interno; las causas son variadas.

GRADO DE INTENSIDAD (cantidad de pérdida auditiva)

1. HIPOACUSIA LEVE: pérdida auditiva entre 20 y 40 decibelios (dB); esta pérdida no ocasiona alteraciones significativas en la adquisición y desarrollo del lenguaje.

2. HIPOACUSIA MEDIA: pérdida auditiva entre 40 y $70 \mathrm{~dB}$; no se percibe la palabra hablada, salvo que ésta sea emitida a una fuerte intensidad; acarrea dificultades para la comprensión y desarrollo del lenguaje; pueden darse problemas de pronunciación y de incorporación de nuevo vocabulario; la comprensión mejora gracias a la lectura labial.

3. HIPOACUSIA SEVERA: pérdida auditiva entre 70 y $90 \mathrm{~dB}$; no se oye la voz, excepto a intensidades muy elevadas; es imprescindible el empleo de audífonos y el apoyo logopédico para llegar a hablar; pueden presentar una entonación monótona, problemas de pronunciación y de incorporación de nuevo vocabulario; pueden percibir el habla a través de la lectura labial.

4. HIPOACUSIA PROFUNDA: pérdida auditiva superior a $90 \mathrm{~dB}$; presentan dificultades para aprender el lenguaje oral. Los audífonos, aunque importantes, aportan mucha menos ayuda que en las anteriores pérdidas; en muchos casos es necesario que estas personas aprendan otro tipo de lenguaje, como el de los signos, para poder comunicarse con los demás.

\section{MOMENTO DE ADQUISICIÓN (cuándo se produjo)}

1. HIPOACUSIA PRELOCUTIVA: es aquella que se produce antes de los 3 años de edad aproximadamente, es decir antes de que haya tenido lugar la adquisición del lenguaje.

2. HIPOACUSIA POSTLOCUTIVA: es la que se produce después de los 4 años aproximadamente, cuando el lenguaje ya se ha adquirido.

ciencias, Discapacidades y Minusvalías (EDDM-86) (Instituto Nacional de Estadística, 1987) supuso un paso adelante en España porque permitió conocer con mayor exactitud los aspectos epidemiológicos de los distintos tipos de deficiencias. Como resultado de esta encuesta, se comprobó que el número global de afectados duplicaba a las anteriores estimaciones de la Administración que eran mucho más bajas. 
Según estos datos, las personas con alguna discapacidad llegaban a 5.7 millones, es decir, constituían el 15\% de la población de España (el 16.4\% de las mujeres y el $13.2 \%$ de los hombres). También es interesante señalar que el porcentaje de discapacidades se incrementa considerablemente en función de la edad:

1. el $62 \%$ de las personas mayores de 65 años;

2. el $23 \%$ de las personas que tienen entre 45 y 65 años;

3. el $4.2 \%$ de las personas entre 15 y 44 años; y

4. el $2.1 \%$ de los menores de 15 años.

Por lo que se refiere a la población en edad laboral (entre 15 y 64 años), las personas con discapacidad representan el $\mathbf{1 0 . 5 \%}$ (2.6 millones de personas).

Si nos centramos en los problemas auditivos, en esta $E D D M-86$ se establece que, en relación con toda la población española, las personas con discapacidades auditivas constituyen un $\mathbf{2 . 3 \%}$ del total (el $2.5 \%$ de las mujeres y el $2.2 \%$ de los hombres). Del total de la población en edad laboral, un 1,51\% tienen dificultades auditivas. Las diferencias significativas en función de la edad y del sexo que se constataron para las discapacidades en general, también se evidencian al analizar los datos relativos a las discapacidades auditivas. Es más significativo el porcentaje de personas con discapacidad auditiva entre 45 y 64 años (el $3,19 \%)$ que el referido al grupo de $15-44$ años (0.65\%). Por otro lado, si se centra el análisis en el tipo de problema auditivo, se comprueba que el porcentaje más reducido se refiere a las personas con problemas de sordera total (el $0,17 \%$ de la población), seguido del de las personas con mala audición $(0,27 \%)$ y finalmente, el de las personas con problemas de sordera en un oído $(0,72 \%)^{4}$. Del total de la población con discapacidades en edad laboral (2.647.001), el 29.7\% corresponde a deficiencias sensoriales; el 13,93\% se refiere a problemas de audición (1,49\% de sordera total; $6,87 \%$ de sordera de un oído; y 6,61\% de mala audición) (Tabla 4).

TABLA 4: Prevalencia de la discapacidad auditiva en la población en edad laboral (15-64 años). Porcentaje en relación con el total de población en cada tramo de edad/sexo. Esquema elaborado a partir de los datos de Pereda y otros (1998).

\begin{tabular}{|l|c|c|c|c|c|c|}
\hline \multirow{2}{*}{ DISCAPACIDAD } & \multicolumn{2}{|c|}{ AMBOS SEXOS } & \multicolumn{2}{c|}{ MUJERES } & \multicolumn{2}{c|}{ HOMBRES } \\
\cline { 2 - 7 } & $\mathbf{1 5 - 4 4}$ & $\mathbf{4 5 - 6 4}$ & $\mathbf{1 5 - 4 4}$ & $\mathbf{4 5 - 6 4}$ & $\mathbf{1 5 - 4 4}$ & $\mathbf{4 5 - 6 4}$ \\
\hline OIR & 0,65 & 3,19 & 0,63 & 3,23 & 0,66 & 3,15 \\
- Sordo total & 0,11 & 0,29 & 0,12 & 0,29 & 0,11 & 0,28 \\
- Sordo de un oído & 0,32 & 1,59 & 0,32 & 1,69 & 0,31 & 1,50 \\
- Mala audición & 0,26 & 1,62 & 0,25 & 1,64 & 0,27 & 1,60 \\
\hline
\end{tabular}

Flórez y otros (1998) al abordar la incidencia del problema de la audición en nuestro país, aluden a datos del INSERSO que indican que en España unas 105.832 personas presentan problemas de audición. De este grupo, unas 28.412 padecen sordera total (el 27\%). En cuanto al origen de estas deficiencias destacan:

4. Estos porcentajes han sido calculados efectuando las medias ponderadas en relación con los datos de la población. 
1. de un $30 \%$ a un $50 \%$ de las deficiencias auditivas son pre y perinatales no genéticas (producidas antes o durante el parto pero no hereditarias);

2. entre un $3 \%$ y $10 \%$ son sorderas postnatales (producidas después del nacimiento);

3. en torno a un $30 \%$ son deficiencias auditivas de causa genética (o hereditaria); y

4. de un $20 \%$ a un $30 \%$ de los casos se desconoce la causa.

El contraste entre las cifras del estudio del INE y el del INSERSO pone en evidencia la falta de acuerdo entre los fuentes, como ya hemos comentado. Es cierto que hay un intervalo temporal importante entre unos datos y otros; que la población de referencia respecto a la que se calculan los porcentajes es diferente en cada caso; y que las categorías de análisis también lo son. Pero nuevamente comprobamos lo difícil que es establecer de forma exacta la incidencia del problema y la necesidad de elaborar, al menos en nuestro contexto, un censo que verdaderamente identifique a las personas con deficiencias auditivas.

\section{La investigación sobre personas con deficiencias auditivas}

La deficiencia auditiva constituye algo más que un simple problema de audición y condiciona en buena medida los procesos de inserción sociolaboral de las personas que la padecen, generalmente de forma muy negativa. $\mathrm{Si}$ a esto añadimos otros problemas que sufren los jóvenes en general en su transición de la escolaridad al empleo, se agravan extremadamente las situaciones a las que se enfrenta este colectivo en sus procesos de normalización e integración laboral, haciéndose cada vez más urgente y necesario que se arbitren medidas efectivas para comenzar a cambiar las dinámicas en las que se ven inmersos.

En este apartado revisamos algunos de los principales trabajos e investigaciones que se han llevado a cabo recientemente, con el objeto de evidenciar y analizar el tipo de problemas y necesidades que las personas con deficiencias auditivas sufren en sus procesos de inserción laboral y otros aspectos relacionados con su desarrollo que nos parecen muy relevantes. Hemos de señalar que muchos de estos estudios se han realizado en otros marcos geográficos, puesto que en España casi no se han planteado durante la última década investigaciones específicas sobre la inserción laboral de las personas con deficiencias auditivas $^{5}$.

El problema radica en que, a las dificultades habituales que conlleva la realización de un estudio riguroso en cualquiera de los campos del desarrollo educativo y social, en el ámbito de la sordera se añaden problemas metodológicos específicos que hacen especialmente difícil la investigación sobre las deficiencias auditivas. En este sentido es importante tener en consideración:

1. La impresionante diversidad de la población con dificultades auditivas: se trata de un colectivo muy heterogéneo, en el que el mismo tipo de problema no produce siempre consecuencias similares. Son tantas las variables implicadas en el desarrollo de las personas con discapacidad auditiva, que lo más ajustado a la realidad es decir que no existen dos personas con deficiencias auditivas iguales.

5. Merece la pena destacar el estudio del GIAEPS (1998) sobre la situación educativa de las personas sordas en Aragón y el trabajo de Alvira y otros (2000) que analiza desde una perspectiva cualitativa las necesidades y demandas de la población con discapacidad auditiva en España. 
2. No contamos con censos actualizados que permitan lograr una idea precisa de las características demográficas de la población con deficiencias auditivas. Como ya hemos dicho, el establecimiento de los aspectos epidemiológicos de este colectivo es muy problemático en todas partes. Es muy difícil establecer con exactitud cuántas personas tienen este problema, su localización y cuáles son sus principales características. Los sistemas empleados siempre presentan algunos problemas y se suele argumentar que es improbable que realmente se refieran a la totalidad de la población con dificultades auditivas.

3. Los anteriores problemas, ligados a la determinación exacta de las características y localización de la población, contribuyen a dificultar los procesos de selección de muestras que representen adecuadamente a la población a la que se refieren.

4. La necesidad de elaborar instrumentos de evaluación específicos adaptados a las características de estos colectivos. El estudio en este campo no permite aplicar instrumentos y técnicas que se utilizan sin dificultad con otros grupos. Por eso los instrumentos empleados en este tipo de trabajos deben ser elaborados específicamente en función de los objetivos de la investigación y también es necesario construirlos teniendo en consideración los condicionamientos comunicativos y cognitivos de las personas con deficiencias auditivas con las que se trabaja.

5. Los procesos de obtención de datos y la aplicación de los instrumentos y técnicas que se utilizan en este tipo de investigación, requieren el establecimiento de procesos de comunicación muy claros y diferenciados de los habituales en otros colectivos. Son muy importantes los problemas de comunicación con las personas sordas, por lo que debe cuidarse especialmente el proceso de obtención de datos y la selección y formación de las personas implicadas en estos procesos.

6. Es necesario vencer las resistencias de las personas con deficiencias auditivas a colaborar en las actividades que se les proponen para poder llevar a cabo las distintas investigaciones. Frecuentemente nos enfrentamos a actitudes recelosas por parte de las personas a las que se les solicita participar o por parte de sus familiares o formadores. Problemas importantes de coordinación entre las instituciones y asociaciones implicadas en el trabajo con personas con deficiencias auditivas, también condicionan negativamente los procesos necesarios para realizar este tipo de proyectos.

No obstante, todas estas dificultades no impiden que se estén llevando a cabo interesantes estudios de acercamiento a la realidad de las personas con deficiencias auditivas. A continuación planteamos de forma muy breve algunos de los aspectos más relevantes de los trabajos e investigaciones realizadas sobre la deficiencia auditiva durante los últimos años, agrupando en seis bloques el contenido de los mismos que estimamos pertinente en relación con el tema que nos ocupa: los procesos de inserción sociolaboral. Los contenidos a considerar son los siguientes:

1. Dificultades asociadas a las deficiencias auditivas;

2. Relevancia de la formación de las personas con deficiencias auditivas;

3. Necesidad de apoyo y orientación para el trabajo;

4. Comunicación e integración en el grupo;

5. Sensibilización de la población ante las deficiencias auditivas;

6. Nuevas tecnologías y deficiencias auditivas. 


\section{Dificultades asociadas a las deficiencias auditivas}

Plantear las dificultades vinculadas a las deficiencias auditivas exige insistir nuevamente en la impresionante diversidad y heterogeneidad de estos colectivos y, por lo tanto, subrayar que es preciso entenderlas sin ninguna pretensión generalizadora. Son muchas las investigaciones y trabajos que se han realizado en todo el mundo, donde se ponen de manifiesto los problemas que viven las personas con deficiencias auditivas. Estas limitaciones, como plantea Warick (1994), afectan a todas las áreas de su desarrollo, particularmente a las de la salud, la educación y el empleo.

Existe una línea de trabajos en los que se analiza la variedad de síntomas asociados a los déficits auditivos, poniendo de manifiesto los problemas específicos de las personas con dificultades auditivas que afectan a las relaciones comunicativas que establecen en los diferentes ámbitos de su desarrollo. El trabajo de Paton (1996) ejemplifica bien este tipo de propuestas al analizar cuáles son los principales síntomas de los adultos con problemas de procesamiento auditivo:

1. hablan y ponen la televisión muy alto;

2. interpretan las palabras demasiado literalmente;

3. necesitan que se les repita lo que se les dice;

4. tienen dificultades al pronunciar las palabras;

5. ignoran a la gente;

6. preguntan más de lo normal para obtener información;

7. confunden palabras con sonidos similares;

8. tienen dificultad para seguir instrucciones en serie;

9. tardan más en comenzar a hablar y lo hacen de forma poco clara;

10. su comunicación es pobre;

11. su memorización es pobre;

12. escuchan mejor cuando pueden observar a quien habla; y

13. tienen problemas de comprensión y comunicación cuando se habla rápido.

Además de este tipo de trabajos que pormenorizan los síntomas asociados, otros se centran en el análisis de alguna de las características más sobresalientes de las personas con deficiencias auditivas. En este sentido por ejemplo, Reiman y otros (1992) identificaron en su investigación como uno de los principales rasgos distintivos de los estudiantes sordos la lentitud en múltiples ámbitos y en base a sus conclusiones elaboraron un programa dirigido a incrementar su rapidez.

Si bien es cierto que estos estudios son de gran interés y contribuyen a incrementar la comprensión de la problemática que nos ocupa, en este momento queremos fijar nuestra atención en los trabajos e investigaciones que se centran en el análisis de las dificultades y problemas de las personas con deficiencias auditivas a un nivel más general, en la medida en que afectan a sus procesos de normalización e integración sociolaboral. Así, uno de los problemas que constantemente aparece en la investigación sobre las personas con deficiencias auditivas de todas las edades y de diferentes contextos, es la discriminación a la que se ven sometidos en su interacción en la comunidad con personas oyentes 
y el aislamiento y marginación en situaciones endogámicas que muchas veces les envuelven. Esta conclusión aparece refrendada en el estudio de Hites (1994), en el que se analizan las múltiples y diferentes situaciones difíciles a las que generalmente tienen que enfrentarse las personas adultas con deficiencias auditivas y en el que también se subrayan los importantes problemas de discriminación social que sufren. Podría pensarse que las políticas a favor de la integración educativa que se han establecido y defendido a nivel general durante los últimos años, estén comenzando a tener un cierto efecto entre las personas más jóvenes de este colectivo, que podrían sentirse menos marginadas con respecto a las personas oyentes que las de las generaciones anteriores. Pero lamentablemente los estudios efectuados con niños y jóvenes sordos continúan indicando situaciones de aislamiento y marginación, pese a su desinstitucionalización de centros específicos y pese a su «supuesta integración educativa» en centros ordinarios.

En un estudio realizado con niños con deficiencias auditivas finlandeses en las etapas de educación infantil y primaria, la mayoría manifestaron tener graves problemas de comunicación. En este trabajo se comprobó que se sienten marginados con un lenguaje minoritario dependiente de signos, por lo que se asocian preferentemente a otras personas sordas y usan a intérpretes para comunicarse con el resto de las personas oyentes (Takala, 1995). Otras investigaciones también demuestran que los niños y adolescentes con deficiencias auditivas se ajustan mejor y prefieren establecer comunicación con compañeros con problemas y niveles de sordera similares a los de ellos. Es decir, incluso en situaciones de integración educativa, tienden a asociarse preferentemente entre ellos (Allen, 1992; Minnett y otros, 1994; Musselmen y otros, 1996; Spencer y otros, 1994).

Si nos centramos en el desarrollo de las personas con deficiencias auditivas con respecto al trabajo, los problemas y dificultades evidenciados por la investigación son múltiples y no pueden desvincularse de las limitaciones ligadas a sus procesos formativos y de otras muchas variables sociales y personales intervinientes. Al analizar las características de los jóvenes con deficiencias auditivas y sus dificultades en el período de transición de la escolaridad al empleo, se constata que es especialmente significativa, en un sentido negativo que no favorece su desarrollo, la marcada dependencia familiar que manifiestan ${ }^{6}$ (Schildroth y otros, 1991). En este mismo trabajo también se comprueba que estos jóvenes tardan más tiempo del normal en graduarse.

Por su parte, Mowry y Anderson (1993) demostraron que las características de personalidad asociadas a la sordera influyen en el proceso y avance hacia el empleo, así como en la posibilidad de conseguirlo y de acomodarse en él. Por ello defienden que es necesario establecer con claridad las implicaciones asociadas a ciertos empleos, los empleos que podrían ser más adecuados para las personas sordas y los servicios de ayuda con los que pueden contar. Lam y otros (1994) también señalan las graves limitaciones a las que tienen que enfrentarse las personas con sordera en sus procesos de integración laboral: los tipos de empleos en los que se las contrata, los salarios que reciben, su poder adquisitivo, su grado de satisfacción con la formación recibida y su grado de satisfacción en el empleo.

6. Muchos otros estudios plantean que la dinámica de sobreprotección de la mayoría de las familias hacia sus hijos sordos contribuye a su retraso en el desarrollo social (Grupo de Investigación y Apoyo a la Educación de las Personas Sordas-GIAEPS-, 1998). 
En esta línea, el trabajo de Kolvitz (1996) establece, partiendo de un análisis longitudinal de varias personas sordas después de 10 años de su graduación que, aunque han llegado a ser económicamente autosuficientes, la mayoría ocupan empleos de muy escasa cualificación o marginales. Otros estudios también evidencian los problemas que surgen en las dinámicas internas en el trabajo, como el de Backenroth (1997) que, al evaluar las dificultades que surgen en el medio laboral, puso de manifiesto que los agrupamientos de personas sordas en el empleo pueden provocar estrés y conflictos de roles. Según sus conclusiones, las experiencias en el lugar del empleo parecen ser más ricas para las personas con deficiencias auditivas que no están agrupadas con otras personas sordas.

Por otra parte, conviene recordar nuevamente que la investigación demuestra que las dificultades que las personas con deficiencias auditivas experimentan en su desarrollo vocacional no son siempre iguales. El-Khiami (1993) por ejemplo, analizó las experiencias vocacionales de 490 personas con problemas auditivos en programas de educación secundaria y comprobó que son muy diferentes dependiendo, entre otros aspectos, de la severidad de la deficiencia auditiva y del tipo de ocupación del que se trate.

Por lo que se refiere a la variable sexo, al igual que la investigación demuestra la discriminación general de la mujer en el mundo laboral, en este campo también se comprueba que las dificultades asociadas a la deficiencia auditiva se hacen aún más graves cuando además se es mujer. En este sentido, el trabajo de McLeod y Gallinger (1992), en el que se hace un seguimiento ocupacional de 4.900 personas sordas que finalizaron sus estudios de bachillerato, constata las discrepancias en las situaciones laborales, ocupaciones y salarios de las mujeres respecto a los hombres. También se demuestra que las mujeres con sordera con un nivel de formación inferior al bachillerato experimentan en mayor medida situaciones de desempleo y subempleo que los hombres con deficiencias auditivas en las mismas condiciones.

En nuestro contexto, particularmente relevantes nos parecen las conclusiones de un trabajo del Grupo de Investigación y Apoyo a la Educación de las Personas Sordas realizado en Aragón para conocer la realidad de las personas con deficiencias auditivas de dicha región (GIAEPS, 1998). Este estudio se efectuó entre 1993 y 1997, trabajando con 103 personas con pérdida auditiva severa y profunda de entre 16 y 35 años. Entre las principales conclusiones de esta investigación, que evidencian las dificultades de la comunidad sorda aragonesa, podemos destacar:

1. La escolarización de los niños con deficiencias auditivas comienza al mismo tiempo que la de los demás niños. Sin embargo, un alto porcentaje tarda más en finalizar su escolaridad obligatoria.

2. La tasa de graduados escolares es similar a la de la población escolar general, pero su nivel educativo es más bajo y manifiestan un bajo nivel de expresión y comprensión escrita.

3. A nivel de educación postobligatoria se comprueban grandes diferencias con la población en general y, normalmente sólo acceden a formación profesional de primer grado.

4. Se muestran muy insatisfechos con la educación recibida, con el profesorado y con las metodologías empleadas (hay que señalar que la mayoría han estado escolarizados en un centro específico para alumnado con deficiencias auditivas). 
5. Existe un elevado porcentaje de abandono al terminar la EGB (67\%). Plantean que faltan estudios adaptados a sus necesidades educativas especiales y su autopercepción es muy negativa con muy escasas expectativas de éxito: baja autoestima, bajo nivel en lecto-escritura y bajo nivel de conocimientos generales.

6. La mayoría no han tenido otros amigos oyentes durante su etapa de escolaridad y sus amigos son otras personas sordas.

7. Su acceso al mundo laboral se ha producido en inferioridad de condiciones respecto a las personas oyentes: sólo un $6 \%$ están empleados en puestos que exigen un nivel de cualificación mínimo.

8. Sus posibilidades de promoción en el empleo están muy limitadas: generalmente se suelen estancar en la misma categoría laboral con la que iniciaron su vida profesional.

9. Les falta autonomía en su desenvolvimiento social en cuestiones burocráticas y administrativas. Encuentran grandes problemas de comunicación al realizar gestiones (en bancos, visitas a médicos, resolución de asuntos legales, etc.). En estas situaciones muchas veces no entienden y les cuesta mucho hacerse entender porque usan un lenguaje distinto al de sus interlocutores.

10. Les gusta mucho la lectura, pero tienen graves dificultades para comprender correctamente lo que leen. Prefieren lecturas con apoyos visuales.

Así pues, son muchos los problemas de formación y de integración sociolaboral que viven las personas con deficiencias auditivas en todas las etapas de su vida. Si bien es cierto que cada situación es única y particular, y que es normal encontrar procesos muy diferenciados, es evidente que existen dificultades y problemas bastante comunes, puesto que los distintos trabajos efectuados en países y en contextos muy diferentes apuntan en direcciones muy similares.

\section{Relevancia de la formación de las personas con deficiencias auditivas}

Todos los trabajos y especialistas coinciden en señalar la importancia fundamental de la educación de las personas con dificultades auditivas para sus procesos de inserción y desarrollo sociolaboral. Muchos de los problemas que viven al integrarse en el mundo del empleo están condicionados por sus limitaciones y dificultades formativas. La educación se considera, por lo tanto, uno de los medios más efectivos e imprescindibles para ayudarles a enfrentarse a estos procesos con los recursos necesarios y en condiciones más positivas. Por esta razón, parece particularmente importante cuidar los programas educativos en los que participa la población con deficiencias auditivas (Congress of the US, 1993). Estos programas son decisivos para su desarrollo personal y social. También, como subraya Schoroedel (1992), los factores escolares son especialmente relevantes para el desarrollo de la carrera (o desarrollo respecto al trabajo) de las personas sordas.

La revisión de los trabajos de distintos autores (Anderson y McGee, 1998; Kolvitz, 1996; Olson, 1992; Reiman y otros (1992); Ryall, 1993; Jaitly y Sims, 1998; Texas Higher Education Coodinating Board, 1994) permite destacar algunos de los objetivos que parece necesario priorizar en los programas de educación de las personas con deficiencias auditivas. Desde el sistema educativo debe ayudarse a las personas sordas a: 
1. Mejorar su capacidad comunicativa.

2. Establecer metas educativas y metas relacionadas con el desarrollo de su carrera.

3. Aprender a vivir independientemente planteándose sus propios objetivos.

4. Adquirir y mejorar sus habilidades sociales

5. Adquirir habilidades de toma de decisiones; $y$

6. Desarrollar habilidades para el trabajo.

Todos los estudios inciden sobre la trascendencia de la educación para el desarrollo vocacional y la inserción laboral de las personas sordas y en la urgencia de vincular los programas educativos con sus necesidades de integración laboral. Es responsabilidad del sistema educativo proporcionarles oportunidades que estimulen al máximo su desarrollo respecto al trabajo. Se plantea la urgencia de ayudarles a adquirir los conocimientos, actitudes, habilidades y recursos necesarios para el desarrollo de su carrera; de proporcionarles experiencias de exploración para que cristalicen sus preferencias vocacionales; de ayudarles a identificar posibles empleos adecuados a sus características particulares; y de prepararlos y apoyar sus procesos de transición de la escolaridad al empleo. Entre las diversas propuestas que en los últimos años se están planteando para estimular el desarrollo respecto al trabajo de estos colectivos, queremos señalar que se están implementando programas muy interesantes dirigidos específicamente a favorecer el desarrollo vocacional de personas con diversos tipos de discapacidad, de diferentes edades y en distintas etapas educativas. Para ejemplificar el tipo de propuestas que se están llevando a cabo, a continuación nos referimos a dos de ellos -el Programa CHOICE y en el PROJECT FOWARD - cuyo eje vertebrador no lo constituye el tipo de discapacidad específica de las personas que participan en ellos (como la deficiencia auditiva, visual, física, etc.), sino el estímulo del desarrollo de las personas en relación con el trabajo dado que su objetivo fundamental es estimular y facilitar la integración sociolaboral de las personas con distintos tipos de dificultades.

A) El programa CHOICE (Career Help at Overbrook for Individuals Who Choose Employment) (Minnesota University, 1998), se centra en la transición de la escuela al empleo de los estudiantes con discapacidad (principalmente con dificultades visuales, pero también con dificultades adicionales, como problemas auditivos, en el desarrollo intelectual, dificultades cognitivas o físicas). Son jóvenes de entre 16 y 21 años que están escolarizados en escuelas públicas. A continuación destacamos algunas de las características y aspectos más significativos de este programa:

1. La organización de experiencias de empleo de verano pagadas para afianzar las habilidades vocacionales que estos jóvenes adquieren durante el curso escolar regular.

2. Los componentes principales del programa son: valoración individual de cada estudiante; desarrollo en el empleo que se ha seleccionado y servicios de apoyo en el empleo.

3. Los tres elementos básicos del programa son: el aprendizaje en la escuela; el aprendizaje en el empleo; y las actividades de conexión entre los aprendizajes que se realizan en la escuela y en el empleo.

4. Para el éxito de este programa es fundamental la colaboración que se desarrolla y mantiene con variedad de empresarios. 
5. A cada estudiante le apoya un profesor que le ayuda a adaptarse al empleo y que le visita regularmente en el lugar de trabajo para observar su progreso.

6. Alumnos y profesores tratan de sensibilizar y educar a los empresarios sobre las discapacidades.

B) El Proyecto FOWARD (Minnesota University, 1997) se viene realizando en un campus universitario desde 1988 con estudiantes con dificultades de aprendizaje, dificultades auditivas, problemas para hablar y con retraso mental de entre 18 y 40 años. A este programa llegan gracias a los planes de transición que estas personas realizaron antes de terminar la secundaria o porque los remiten desde distintos tipos de programas de post-secundaria. El objetivo principal que se persigue es educar sus habilidades vocacionales y proporcionarles experiencias de trabajo para estimular el desarrollo de su carrera.

1. Entre las actividades que realizan destacan:

- Identifican las metas u objetivos de los estudiantes.

- Aplican inventarios de intereses vocacionales.

- Valoración vocacional y de sus aptitudes y estilos de aprendizaje.

- Los estudiantes y profesores eligen un empleo y desarrollan experiencias de trabajo cooperativo.

- Los estudiantes, formadores, coordinadores y otros participantes en el programa reciben informes escritos y participan en conferencias individuales al final de cada semestre.

- Los estudiantes se gradúan en dos años.

2. Los componentes más importantes de este programa son: la valoración de habilidades; las experiencias de trabajo cooperativo; la colaboración; y el desarrollo de actividades de conexión entre educación y empleo.

3. El éxito de este programa se basa en estos factores:

- El marco universitario en el que se desarrolla proporciona a las personas con discapacidades que participan en el programa oportunidades para relacionarse con iguales, posibilitando la integración en un contexto normalizado.

- Se establece un sistema de créditos que se consiguen en función de los objetivos alcanzados.

- Se les proporcionan experiencias de trabajo.

- Participan en prácticas en el empleo en contextos laborales reales.

- Colaboración profesional entre educadores y empresarios.

- Dedicación del personal que trabaja en este proyecto.

Por otra parte, también se están desarrollando programas educativos para personas con deficiencias auditivas diseñados específicamente para ellas y que también priorizan el objetivo de su preparación para el trabajo ${ }^{7}$. Dabbs (1991) por ejemplo, nos remite a una experiencia de estas características que se está llevando a cabo en una escuela americana para

7. Schoroedel (1992) propone el desarrollo de una red nacional de educación para la carrera específicamente dedicada a este colectivo para favorecer su desarrollo respecto al trabajo. 
estudiantes sordos con múltiples handicaps o deficiencias asociadas. En este centro están desarrollando un programa de preparación para el empleo que combina el trabajo en las distintas áreas curriculares durante la mitad de la semana (lenguaje, matemáticas, habilidades sociales y conocimientos científicos), con experiencias en el empleo durante el resto de la semana supervisadas por el profesor.

Partiendo de las experiencias formativas que se están llevando a cabo en diferentes contextos con personas con deficiencias auditivas destacamos algunas de las consideraciones que deben tenerse en cuenta al trabajar con este colectivo:

1. Es necesario ser especialmente cuidadosos con las dinámicas relacionales generadas en los programas de formación de personas sordas. En los centros y programas de formación para estos colectivos se deben emplear métodos de comunicación adecuados que favorezcan la comunicación en otros ámbitos (Dabbs, 1991; Gregory, 1992).

2. Es imprescindible para fomentar la inserción sociolaboral de las personas con dificultades auditivas, vincular más estrechamente la formación que se les proporciona desde el sistema educativo con el mundo laboral. Debe relacionarse dicha formación con las funciones y actividades que se desarrollan en el mundo laboral. Esto exige una estrecha colaboración entre el sistema educativo y el mundo laboral (Nathanson, 1991). La implicación y coordinación entre los responsables del programa, el profesorado y los representantes del mundo sociolaboral y empresarios de la comunidad en la que se establece es uno de los factores más importantes para el éxito del programa.

3. Los procesos de diagnóstico y evaluación individualizados de cada uno de los estudiantes con deficiencias auditivas (valoración vocacional, de aptitudes, de intereses, de estilos de aprendizaje, de habilidades...) son particularmente relevantes para estimular su desarrollo. También es fundamental organizar servicios de seguimiento de los estudiantes con déficits auditivos (Minnesota University, 1997, 1998).

4. Cada estudiante con discapacidad auditiva debe establecer su propio plan de carrera individualizado. La identificación de las metas y objetivos de cada estudiante es una de las claves de cualquier buen programa que trate de favorecer el desarrollo respecto al trabajo de las personas con deficiencias auditivas (Minnesota University, 1997). En este sentido, Woodrick y Wolfe (1995) subrayan que los jóvenes con deficiencias auditivas que tienen una meta de carrera definida claramente es más probable que completen su educación post-secundaria. Por otra parte, también suele destacarse la importancia de que se planteen metas para un buen rendimiento en el mundo productivo (Schildroth y otros, 1991).

5. Es necesario y urgente organizar programas de secundaria que tengan en cuenta las necesidades de desarrollo respecto al trabajo de las personas con deficiencias auditivas (Schoroedel, 1992). Es responsabilidad de las instituciones encargadas de la formación de estos colectivos el planificar y crear estrategias que estimulen el desarrollo de sus carreras (Texas Higher Education Coordinating Board, 1994). Se recomienda a los estudiantes con deficiencias auditivas, que seleccionen las instituciones post-secundarias que proporcionen servicios de apoyo y orientación para responder a sus necesidades específicas (Woodrick y Wolfe, 1995). 
6. Uno de los aspectos más relevantes que condiciona en buena medida el desarrollo del programa, es la formación y competencia profesional de los profesionales implicados. Hay un consenso generalizado que subraya la importancia de que mantengan buenas relaciones entre ellos, así como de que sean especialmente sensibles a las dificultades y problemáticas asociadas a la deficiencia auditiva. Kolvitz (1996), al estudiar el desarrollo profesional y administrativo del personal de secundaria que trabaja con personas sordas, plantea la necesidad de que los objetivos educativos sean muy claros; de que haya una buena relación entre el profesor y el intérprete y entre el alumno y el intérprete; de que se definan muy claramente los papeles de los servicios para personas con discapacidad; de que se utilicen estrategias de motivación adecuadas; y de sensibilizar a la población sobre la diversidad cultural.

7. Otro factor fundamental que debe ser tenido muy en cuenta en los programas educativos, es la decisiva influencia que ejerce la familia en el desarrollo con respecto al trabajo de sus hijos con deficiencias auditivas (Schoroedel, 1992). Se recomienda especialmente aprovechar su potencial formativo implicándolos en su desarrollo y en los programas de formación de forma muy activa y comprometida.

Por otra parte, también queremos destacar que unido al planteamiento de todo este tipo de programas formativos de personas con deficiencias auditivas, otra de las líneas de trabajo se centra en la elaboración de materiales educativos complementarios y de apoyo a estos programas educativos, con la finalidad también de estimular y favorecer al máximo su desarrollo. El tipo de materiales que se elaboran es muy diverso, desde los más sencillos y habituales empleados en las aulas, hasta los más complejos y sofisticados generados por el avance de las nuevas tecnologías también en este campo: cuadernos para el alumno, cuadernos para el profesor o tutor, libros, cuentos, posters, videos, programas de ordenador interactivos, CD.ROMs, etc.

Así, Woodrick y Wolfe (1995) por ejemplo, elaboraron 55 perfiles de personas con dificultades auditivas que han tenido éxito. Los presentan analizando el tiempo que hace que sufren la pérdida auditiva y la causa, la formación académica que han recibido y el campo ocupacional en el que los han empleado. Con este material tratan de ofrecer modelos de roles exitosos y lo han diseñado para poder ayudar en los procesos de exploración de la carrera de los estudiantes con dificultades auditivas. También es un recurso que puede ser utilizado en el currículum de lengua como material de lectura y puede permitir discusiones en grupo sobre la universidad y sobre las opciones de carrera de estos jóvenes con dificultades auditivas. Otro ejemplo es el de la Batería de Competencias de Transición para adolescentes y adultos jóvenes con deficiencias auditivas elaborada por Bullis y Reiman (1992), con el objeto de ayudarles a proponerse metas y en la búsqueda de empleo.

Entre los materiales diseñados para trabajar con personas sordas vinculados al avance de las nuevas tecnologías durante los últimos años, podemos aludir al trabajo de Hutchins (1996) que combina la presentación de un CD-ROM y de los materiales educativos asociados. Contiene un libro para niños muy conocido desde hace 30 años, en el lenguaje de signos americano y en inglés signado, y va acompañado de texto, gráficos, animaciones y voz para que pueda ser utilizado tanto por niños oyentes, como por niños con deficiencias auditivas. También presenta 120 películas muy cortas en lenguaje de los signos y una selección de juegos para reforzar los conceptos y vocabulario que se trabajan en el libro. En resumen, la principal idea que queremos destacar es la proliferación de materiales de apoyo para los 
programas de educación y de orientación en general, y de educación y orientación para el trabajo en particular, para las personas con deficiencias auditivas, lo cual parece indicar que el desarrollo de esta línea de trabajo no ha hecho más que empezar.

\section{Necesidad de apoyo y orientación para el trabajo}

Como hemos visto, las necesidades de las personas con deficiencias auditivas en sus procesos de desarrollo e integración sociolaboral son muchas y muy diversas. Todos los trabajos, especialistas, formadores y familiares de personas con deficiencias auditivas coinciden en señalar la urgencia y relevancia de organizar y desarrollar servicios y programas de orientación de calidad que les ayuden a superar los diferentes obstáculos y retos a los que se enfrentan a lo largo de su vida (Gateway Technical Institute, 1993; GIAEPE, 1998; Kolvitz, 1996; Long y Clarck, 1993; Minnesota University, 1997). Esta necesidad de apoyo y ayuda orientadora es una constante reivindicación para la formación y atención a todos los colectivos con discapacidad, en todas las etapas de su desarrollo a lo largo de la vida. En Wisconsin por ejemplo, se considera que el éxito de la educación de los estudiantes adultos con discapacidad (una o varias) viene en gran medida condicionado por la calidad de los servicios de apoyo y orientación que se establezcan. Desde estos programas se trabaja especialmente con el profesorado para ayudarle a entender las implicaciones educativas particulares de cada discapacidad (Gateway Technical Institute, 1993). En otras investigaciones recientes, también se destaca la importancia de orientar y apoyar a los padres de niños con dificultades auditivas (Hintermair, 2000).

Ante las necesidades de los colectivos de personas con discapacidad, la Administración tiene un importante reto que afrontar instrumentando las medidas políticas y legales que hagan posible el establecimiento del tipo de apoyos y ayudas que son necesarios. En un reciente trabajo, Busby y Daneck (1998) señalan la importancia que han tenido los cambios que se han producido en la política y en la legislación para hacer posible los servicios de ayuda en la transición de la escolaridad al empleo que se están desarrollando con éxito actualmente con estudiantes con discapacidad en Estados Unidos ${ }^{8}$. Por lo tanto, responder a las necesidades de orientación detectadas en el sentido en el que apuntan los planteamientos más innovadores en este campo, exige no sólo mucho esfuerzo y trabajo, sino también que las autoridades competentes adopten las medidas oportunas para fomentar y estimular el desarrollo de programas de orientación.

Durante las últimas décadas la orientación para el desarrollo de la carrera (es decir, la orientación para el desarrollo de las personas respecto al trabajo) ha adquirido una espectacular fuerza motivada por las urgentes necesidades de desarrollo vocacional de todas las personas que se ligan a los incesantes cambios del mundo sociolaboral. La necesidad de recibir apoyo y orientación con respecto al trabajo, es un derecho de toda persona en cada una de las etapas de su desarrollo, como establece la NCDA (National Career Development Association, 1993) al consensuar los principios que sirven de base para la elaboración de programas de educación y orientación para el trabajo. También es uno de los derechos fundamentales de las personas más desfavorecidas y que viven situaciones de mayor discrimina-

8. Se refieren específicamente a la legislación de 1992 de los Amendements to the Rehabilitation Act, The Individuals with Disabilities Education Act y School to Work Opportunities Act. 
ción laboral. Entre estos colectivos, el de las personas con deficiencias auditivas requiere particular atención por la relevancia y gravedad de sus necesidades de desarrollo respecto al trabajo.

En Canadá, por ejemplo, Mason (1997) analiza las experiencias de transición al mundo laboral de 50 personas con deficiencias auditivas y destaca la importancia de organizar programas de apoyo y orientación a la transición, tanto desde dentro de la escuela, como desde el mundo laboral. Otro interesante ejemplo de orientación en el ámbito escolar, lo refieren Downie y Moore (1998) en una reciente publicación en la que destacan la importancia de los programas de educación para la carrera ${ }^{9}$ que se están desarrollando en una escuela para personas sordas californiana. En este centro han optado por centrar especialmente sus programas educativos en el estímulo del desarrollo respecto al trabajo de la población con deficiencias auditivas a la que atienden, vinculando los contenidos curriculares de las distintas áreas al mundo laboral. Las principales claves de estos programas son:

1. proporcionar asesoramiento individualizado en relación con la carrera ${ }^{10}$,

2. organizar actividades de transición para los estudiantes;

3. formar a los estudiantes para que realicen su propio «plan individualizado de transición»; y

4. estimular a padres/madres y profesores a que se impliquen en la planificación de la transición de sus hijos y alumnos.

Finalmente, no debemos olvidar la relevancia de establecer también servicios y programas adecuados de orientación y apoyo en el empleo, para responder a las necesidades de estos colectivos desde el mismo medio laboral. Kolvitz (1996) argumenta que la supervisión, el seguimiento y los servicios de apoyo, son las claves esenciales para el desarrollo profesional y mantenimiento en el empleo de las personas con deficiencias auditivas.

\section{Comunicación e integración}

La comunidad de personas sordas se caracteriza no sólo por la deficiencia auditiva, sino también por una cultura y comunicación propia y particular. El siguiente texto lo ejemplifica de forma precisa:

«Si se define cultura como herencia, lenguaje y un conjunto de costumbres y valores compartidos por sus miembros y transmitidos de generación en generación, entonces la comunidad de las personas sordas verdaderamente constituye una cultura»(Gilliam y Easterbrooks, 1997).

Por ello, uno de los problemas más importantes de las personas con deficiencias auditivas lo constituye el establecimiento de procesos de comunicación adecuados con los que les rodean. Las dificultades de comunicación entre personas con déficits auditivos y personas

9. Con los programas de educación para la carrera se pretende estimular al máximo del desarrollo de las personas respecto al trabajo a través de: 1) la vinculación de los contenidos curriculares habituales que se trabajan en un campo con los contenidos necesarios para el desarrollo vocacional; y 2) de la colaboración comunitaria.

10. En el sentido consensuado actualmente que por carrera entiende la totalidad de trabajos pagados y no pagados que la persona realiza a lo largo de su vida $(N C D A, 1993)$. 
oyentes han supuesto a la largo de los años una poderosa barrera a la integración normalizada de estos colectivos. La complejidad del tema y la dificultad de resolverlo se hace más evidente al considerar la gran pluralidad y diversidad de situaciones asociadas a las deficiencias auditivas.

La polémica se centró durante muchos años sobre cuál debería de ser el lenguaje que se utilice en la educación de las personas sordas (lenguaje de signos o lenguaje oral), esgrimiéndose razones de peso a favor de ambas posturas. La principal crítica que se hacía a la enseñanza del lenguaje de signos, era que estos niños adquirirían así un lenguaje minoritario que podía aislarlos y segregarlos más, haciendo muy difícil su comunicación con el resto de la población. También se consideraba su posible interferencia en el aprendizaje del lenguaje oral que todos utilizamos. Sin embargo, en relación con esta polémica, son muy clarificadores los argumentos que se plantean en un interesante trabajo publicado en España sobre las personas sordas (GIAEPS, 1998):

«... la adquisición e interiorización de un código lingüístico por los niños sordos es un factor fundamental, tanto para su comunicación e interacción social, como para su propio desarrollo cognitivo. Este código lingüístico puede ofrecerlo el lenguaje oral, pero también -y con mayor facilidad para su adquisición- la lengua de signos, en el caso de los niños sordos. Por eso se considera importante que los niños sordos pequeños adquieran la lengua de signos en unos años, de los dos a los seis, en los que existen severas dificultades para interiorizar el lenguaje oral. Los estudios realizados comparando el desarrollo lingüístico y cognitivo de niños sordos que aprendieron en edades tempranas el lenguaje de signos, con niños sordos que no lo adquirieron, confirman la importancia de la comunicación signada en los primeros años.»

«... la época que abarca desde el nacimiento hasta aproximadamente los seis años, constituye un período crítico para la adquisición del lenguaje. En el caso de los sordos, parece imprescindible proporcionarles en ese período una estimulación lingüística que permita el desarrollo de las estructuras neurológicas implicadas en el lenguaje. Ya que la estimulación oral resulta muy difícil en estos niños, es preciso considerar la utilización del lenguaje de signos. El desarrollo neurológico así obtenido puede aumentar el potencial para la utilización posterior del lenguaje oral.»

La conclusión más clara de este estudio es la necesidad de una educación bilingüe de las personas sordas, que fomente la adquisición de la lengua de signos como su lengua natural y que facilite el aprendizaje de la lengua oral por ser la lengua que mayoritariamente se usa en la sociedad. Parece pues que sería conveniente establecer con las personas con deficiencias auditivas una lengua de signos inicial, que le sirva de base para el aprendizaje del lenguaje oral, para poder llegar a ser «bilingüe» (lo que en España se conoce como «bilingüismo sucesivo») $)^{11}$.

Actualmente, el reconocimiento de la importancia y del valor de la lengua de signos para la comunidad de personas con deficiencias auditivas se está afianzando cada vez más. Ejemplo de ello es el impulso a actuaciones y programas en este sentido, como el de EUD (Programa Europeo de Lenguaje de Signos) que trata de potenciar la formación, utilización

11. Otra tendencia cada vez más arraigada es la que se conoce como «comunicación total» y pretende ofrecer a cada persona la modalidad de comunicación que necesite en cada momento, con el fin de estimular su óptimo desarrollo posible. 
y fomento de la lengua de signos para que las personas con deficiencias auditivas tengan acceso al empleo, comunicación, formación y participación plena sin discriminaciones ni barreras (IMSERSO, 1999) $)^{12}$.

Por otra parte, debemos señalar en relación con la lengua de signos que, aunque se han hecho considerables progresos hasta la fecha, todavía se plantean importantes retos relativos a su unificación geográfica (existe un lenguaje de signos referido a cada contexto geográfico y lingüístico) ${ }^{13}$, el establecimiento de una gramática clara, la ampliación de vocabulario, etc. Además debemos de señalar que, incluso tratándose de un mismo contexto como el europeo, las diferencias en cuanto a la utilización, extensión y relevancia concedida a este tema en cada país son muy significativas. Schulte (1995) por ejemplo, analizando la educación superior de las personas sordas en Alemania constató que, aunque no existen universidades especiales, sí se realizan numerosas adaptaciones para responder a las necesidades de las personas con deficiencias auditivas y que se dispone de la ayuda de intérpretes para estos casos. En España por el contrario, no contamos con ningún programa similar y pensar en la posible utilización del lenguaje de signos para responder a las necesidades comunicativas de estos colectivos en la mayoría de nuestras universidades aún sigue siendo una posibilidad remota.

En España, en la actualidad, la tendencia más clara también promueve el bilingüismo sucesivo. Desde el punto de vista normativo, todavía no se ha reconocido oficialmente una Lengua de Signos Española (L.S.E.), aunque han sido numerosas las iniciativas de diversos grupos parlamentarios apoyando tal reconocimiento. Una de las más relevantes fue la del 16 de Diciembre de 1997, cuando la Comisión de Política Social y Empleo del Congreso aprobó una Proposición no de Ley instando al Gobierno a elaborar un informe. Posteriormente, el 13 de Abril de 1999, el Senado aprobó una propuesta de modificación instando al Gobierno a intensificar el desarrollo de la lengua de signos como fundamental para el colectivo de personas sordas (Resa, 1999).

Por otro lado, en relación con el tema del lenguaje y la comunicación, frecuentemente se plantea cuál es el mejor sistema de educación para las personas con deficiencias auditivas: se trata de decidir si es más conveniente integrar a las personas sordas en centros ordinarios o es preferible que se les eduque en centros especiales para personas con deficiencias auditivas. Esta cuestión en términos generales, no parece aún concluida ni resuelta, por lo que la controversia está servida y en cada marco y contexto se toman opciones distintas.

A este respecto, Gilliam y Easterbrooks (1997) aluden al problema del aislamiento demográfico que muchas de estas personas sufren y a que la escasa convivencia con iguales muchas veces les genera un notable desarraigo cultural. Por eso en su trabajo plantean los beneficios de asistir a escuelas residenciales para alumnos con deficiencias auditivas, que incluyen programas académicos comprensivos, programas de salud y de socializa-

12. El EUD publica una revista en la que da a conocer sus actuaciones. El grupo nacional del $E U D$, integrado por expertos y usuarios, tiene representación del Consejo Nacional de Sordos, FIAPAS (Federación Nacional de Padres y Amigos de Sordos) y profesionales. El CEAPAT (Sociedad Internacional de Comunicación Aumentativa y Alternativa) participa en calidad de experto en este grupo.

13. Mackie (1993) realizó un estudio de observación de las dificultades de las personas con sordera profunda. Entre sus conclusiones destacó que si se lograse desarrollar un verdadero Lenguaje de Signos Universal se contribuiría en gran medida a unir la cultura de las personas sordas y la de las oyentes. 
ción. La mayoría de estos centros en Estados Unidos están trabajando con edades que van desde la educación infantil hasta los 12 años y cuentan con personal especializado que puede comunicarse con fluidez con los estudiantes en todas las áreas. Entre las ventajas de este tipo de instituciones señalan la libertad de comunicación que sienten los niños y jóvenes con deficiencias auditivas moviéndose en estos contextos con iguales con el mismo tipo de problemas y sistemas de comunicación; el sentido de pertenencia que desarrollan; la posibilidad de participar en deportes y otras actividades en grupo; y las oportunidades que se les ofrecen de tener experiencias en las que tengan éxito y salgan airosos. Entre los inconvenientes de estos centros, destacan el hecho de estar más lejos de la familia que no participa tanto en su educación; la mayor dificultad para adaptarse a la vida adulta porque se ha aprendido a depender y se ha tenido un contacto más limitado con la comunidad más amplia; y que el currículum suele ser menos riguroso que en otras escuelas. La decisión, concluyen, debe tomarse teniendo en cuenta las necesidades educativas y emocionales de cada alumno, sopesando cuidadosamente los pros y los contras y teniendo en cuenta sus intereses.

En Canadá la discusión también parece centrarse tanto en el tema del bilingüismo como en el del biculturalismo, y se está poniendo especial énfasis en las grandes posibilidades de las escuelas para personas con deficiencias auditivas. Mason (1994) argumenta que, aunque se opta por una educación bilingüe y bicultural, la tendencia más significativa en Canadá, Suiza y Estados Unidos se decanta hacia los centros específicos. También subraya que se están analizando las implicaciones de este modelo para la familia y las universidades.

Por lo que se refiere a las ventajas y desventajas de la integración en contextos educativos normalizados, la cuestión aún sigue siendo muy controvertida y los efectos que estos procesos integradores están teniendo es necesario analizarlos en cada caso particular. En un reciente trabajo, Innes y Nowel (1997) investigaron la integración de niños con deficiencias auditivas en escuelas públicas ordinarias. Entre los beneficios de su integración señalaron: la posibilidad de vivir en sus hogares; las oportunidades para comunicarse con el mundo oyente; la posibilidad de aprender los standars de las personas oyentes; y la disponibilidad de participar en un mayor número de programas académicos y vocacionales. Entre las limitaciones de la integración se destacan ${ }^{14}$ : el potencial aislamiento de los profesores y compañeros; el tener menos oportunidades de educación directa o individualizada; y la menor interacción con iguales y con profesionales especializados en educación de personas sordas.

Por lo que se refiere al contexto en el que trabajamos, la clara opción que desde la Administración se ha tomado a favor de una política integradora de las personas con discapacidad auditiva en los centros ordinarios, ha llevado a una integración «al menos física» de estos colectivos en las instituciones educativas generales. No obstante, sus efectos son más dudosos como se puede constatar en las conclusiones del estudio empírico que hemos realizado para evaluar las necesidades y problemas de los jóvenes con problemas auditivos en sus procesos de integración sociolaboral.

14. Desde otros contextos también se subrayan los problemas y limitaciones de la integración de las personas con deficiencia auditiva en las escuelas ordinarias. Cohen (1995) por ejemplo, señala que en Israel la experiencia de integrar a niños sordos en escuelas normales ha resultado ser negativa, que la comunicación entre personas oyentes y sordas es muy poca y que no se ha producido una verdadera asimilación social. 


\section{Sensibilización de la población ante las deficiencias auditivas}

Son muchos los estereotipos que durante años se han atribuido a las personas con deficiencias auditivas, como el de que una de sus principales características es que son desconfiados o el de que su rendimiento en actividades manuales es muy elevado. Como sucede con otros tipos de estereotipos, se trata de preconcepciones falsas en las que se atribuye a los miembros de un colectivo una característica determinada, por su mera pertenencia a dicho grupo. Como hemos señalado en varias ocasiones, la deficiencia auditiva es el aspecto común que comparte este colectivo, siempre con tipos de problemas, disfunciones y consecuencias muy distintas en cada caso. Podemos decir que la gran heterogeneidad y diversidad que caracteriza a las personas sordas es uno de sus rasgos más sobresalientes, por ello esas visiones estereotipadas y cargadas de prejuicios, que condicionan la forma de percibirlos y de relacionarse con ellos, son radicalmente falsas.

De ahí que la sensibilización de la población en general ante las deficiencias auditivas supone otro de los retos prioritarios que tenemos que abordar para generar los cambios positivos necesarios. Algunos de los trabajos que recientemente se han hecho en esta línea son muy interesantes. Cambra (1996) realizó un estudio con 222 estudiantes universitarios a los que presentó diversos descriptores de personalidad para que se los aplicaran a personas con deficiencias auditivas, visuales y sensoriales. Sobre las personas sordas comprobó que hay dos estereotipos muy extendidos y afianzados: los consideran personas poco sociables y también piensan que su inteligencia es limitada. Por otra parte, también pudo constatar que aquellas personas que ya conocían a alguna persona sorda les atribuían descriptores de personalidad más positivos.

En otro proyecto desarrollado durante el mismo año se demuestra que esa atribución a las personas sordas de inteligencia limitada es errónea. Resulta también muy interesante porque, entre otros aspectos, trata de desarrollar una mayor sensibilidad cultural ante la deficiencia auditiva. En este trabajo de Vialle y Paterson (1996), se trata de proporcionar las oportunidades necesarias para estimular el potencial de las personas sordas que tienen un desarrollo intelectual extraordinario. Se plantea que, durante demasiado tiempo, se ha concedido excesiva importancia a la facilidad lingüística en la identificación de las personas con altas capacidades intelectuales. Como resultado, han sido identificadas como «superdotadas» menos personas con deficiencias auditivas de las que verdaderamente hay. En este estudio se analiza la sordera como una diferencia cultural y se presentan también casos que subrayan experiencias educativas y personales de individuos con deficiencias auditivas cuyos logros como adultos los clasificarían como «superdotados».

Las personas sordas con un elevado desarrollo intelectual con las que se trabaja, enfatizan que la «superdotación» no es un potencial innato, sino que es el resultado de las oportunidades que han tenido, comparadas con las de otras personas sordas. También reconocen la importancia de un ambiente familiar que les apoye para ayudarlos a lograr esos niveles de rendimiento (ratificando nuevamente la relevancia de los programas de formación y orientación y del papel de la familia en el desarrollo de las personas con deficiencias auditivas a los que antes nos referíamos). Por último, otro aspecto de gran interés, es que como factor crítico de su éxito también se destaca su identificación con la comunidad de personas con deficiencias auditivas.

Otros estudios recientes en los que también se ha trabajado tratando de sensibilizar a la población sobre la deficiencias auditivas, se plantean objetivos muy diferentes, aunque 
siempre tratando de generar y extender esos cambios positivos de forma más general en la sociedad. Klee (1994) describe un programa desarrollado por el Gobierno de Kentucky en colaboración con la Universidad, para formar en el lenguaje de los signos americano ( $A S L)$ a empleados oyentes (funcionarios). El objetivo de este programa es sensibilizarlos ante la deficiencias auditivas y conseguir que se puedan comunicar de forma más efectiva con las personas sordas.

Por su parte, Zahn y Kelly (1995) trabajan con una muestra de 155 estudiantes universitarios, a los que presentan un vídeo mostrándoles imágenes positivas de personas con distintos tipos de deficiencias auditivas trabajando en 8 ocupaciones diferentes. Las pruebas pre-test y post-test que efectuaron evidencian que se producen cambios positivos en la actitud de estos estudiantes y que ese cambio de actitud se mantiene cuando se les aplica un post-test treinta días más tarde, además de generalizarse para otras ocupaciones. De esta forma demuestran, que es posible cambiar las actitudes extendidas socialmente y que se puede formar a los futuros trabajadores y empresarios sensibilizando y generando actitudes más positivas sobre las personas sordas en el empleo.

Todas estas experiencias demuestran que la lucha contra los estereotipos sociales y la sensibilización general de la población ante la deficiencia auditiva es uno de los retos más importantes que la educación tiene planteados en este campo ya que puede cambiar la imagen de este colectivo y, en consecuencia, incrementar sus posibilidades de integración social y laboral.

\section{Nuevas tecnologías y deficiencias auditivas}

El avance de las nuevas tecnologías en todos los campos ha sido espectacular durante las últimas décadas y la relevancia de estos avances tecnológicos y la magnitud de sus implicaciones para las personas con dificultades sensoriales, psíquicas y físicas se enfatiza constantemente en todo el mundo. Autores como Galvin y Scherer (1996) destacan la importancia que está teniendo este desarrollo tecnológico en el apoyo a las personas con todo tipo de discapacidad. Thomson y otros (1998) plantean que las tecnologías pueden mejorar y ampliar en gran medida la carrera académica y las oportunidades de empleo de los estudiantes con discapacidad ${ }^{15}$.

También el desarrollo sin precedentes de las tecnologías en las últimas décadas ha contribuido a mejorar la calidad de vida y la comunicación de las personas con deficiencias auditivas. A lo largo de los años, las tecnologías destinadas a aprovechar los restos auditivos, a compensar la falta de audición por medio de luces y a facilitar la comunicación, han ido evolucionando, generando audífonos mejores y más ligeros y sistemas cada vez más sofisticados y adaptados a los diversos problemas auditivos. Gracias a todas ellas (fax, DTS, móviles vibratorios, luminotecnia...), las importantes barreras comunicativas de las personas sordas han comenzado a ser menores y también se ha facilitado considerablemente su acceso a la información.

15. Elaboran una guía para profesionales de la educación y del empleo que trabajan con estudiantes con dificultades, para enseñarles las formas en las que pueden utilizar las tecnologías. 
A continuación recordamos algunos de los avances tecnológicos más significativos en relación con el mundo de las deficiencias auditivas partiendo de la información contenida en un Dossier elaborado por FIAPAS recopilando los problemas y soluciones que se le plantean a las personas sordas en sus relaciones de comunicación:

1. Prótesis auditivas: audífonos convencionales, retroarticulares, gafas auditivas, intraauriculares, intracanales, vibrador óseo, implante coclear.

2. Ayudas técnicas en la escuela: audífono, equipos de reeducación auditiva, ayudas técnicas dirigidas a favorecer la estimulación vibrotáctil, ayudas técnicas que acercan la señal auditiva, como equipos colectivos (SUVAG) o equipos individuales de FM, ayudas técnicas que ofrecen información visual de los sonidos o información espectográfica de la voz humana, diversos materiales informáticos, videoconferencias.

3. Ayudas técnicas en el hogar: timbres luminosos para la puerta y el teléfono, despertadores luminosos, vibratorios y combinados, intercomunicadores, amplificadores de sonido para el teléfono, televisiones y equipos de músicas con sistemas de amplificación con cable o sin cable, estos últimos puede utilizar sistemas de infrarrojos o sistemas de inducción magnética, sistemas combinados y multiusos a través de señales luminosas.

4. Ayudas técnicas para la comunicación: teléfonos de texto (en España el TELESCRIT 1005 y el VIDEOTEX B/N 200), teletexto, medios técnicos e informáticos utilizados normalmente por la generalidad de la población oyente y sorda, como fax, correo electrónico, internet.

El desarrollo tecnológico ha afectado a todos los campos relacionados con las deficiencias auditivas: en la medicina, respondiendo al déficit orgánico a través de mejores sistemas de tratamiento y de intervención quirúrgica y generando y perfeccionando prótesis auditivas cada vez más sofisticadas y de mayor calidad; en el campo educativo, facilitando las adaptaciones del medio necesarias a las características de estos colectivos y estimulado la elaboración de todo tipo de materiales, como hemos visto, muy útiles en el desarrollo de programas; en el hogar, ayudando a adaptar el contexto donde la persona se desenvuelve en su vida cotidiana a sus limitaciones y posibilidades; y también en el medio laboral y en general en todos los marcos formativos y sociales, posibilitando el desarrollo y uso de instrumentos técnicos para ayudarles a superar las barreras comunicativas.

DeGraw y Stroud (1993) plantean que en nuestros días todos los estudiantes necesitan habilidades para manejar los ordenadores y que para los estudiantes con discapacidades son incluso más importantes, porque pueden constituir la única puerta abierta a las habilidades que exige el mercado laboral. Para muchos estudiantes pueden ser el mecanismo que les permita participar de forma más completa en el proceso educativo, aprender las habilidades requeridas en el desarrollo de su carrera, y adaptarse al mundo del trabajo. Por ejemplo, Stewart (1996) se refiere al desarrollo de un Comunicador Personal en CD-ROM, que permite el establecimiento de interacciones de los jóvenes con deficiencias auditivas. En estos programas se incluyen videoconferencias, diccionarios, libro de notas, conversaciones grabadas y lenguaje cotidiano. El mismo autor presenta un prototipo de un sistema de vídeo interactivo que enseña una comunicación signada que puede ser muy útil en la educación de personas con deficiencias auditivas. También DeGraw y Stroud (1993) recomiendan el uso de un programa de ordenador que además de facilitar la comunicación, permite reemplazar 
palabras por iconos visuales y puede servir de acceso a muchos procesos educativos y programas de formación para el trabajo. Por otro lado, además de los programas que se utilizan en la enseñanza presencial, también en este campo se considera que las videoconferencias son la esperanza en la comunicación a distancia, para permitir el continuo enriquecimiento y la expansión de la lengua de signos.

Sin embargo, pese los importantes avances que se han producido en este sentido, tenemos que señalar que siempre están condicionados a las posibilidades reales del contexto en el que vive la persona con deficiencia auditiva ${ }^{16}$. Como muy bien plantea Tucker (1993), para que realmente las nuevas tecnologías constituyan un verdadero avance social y permitan la supresión de las barreras de comunicación, deben ser incluidas en el empleo y en la comunidad y es necesaria una mayor participación social. Indiscutiblemente, como sucede a nivel general, el acceso a las nuevas tecnologías de las personas con deficiencias auditivas seguirá siendo una cuestión discriminatoria mientras siga estando vinculado y condicionado por el poder adquisitivo del grupo social al que pertenezcan.

\section{Recomendaciones a tener en cuenta al formar o emplear a personas con deficiencias auditivas}

Cualquier tipo de programa destinado a personas con dificultades auditivas exige que nos adaptemos a sus características y necesidades. El éxito de toda estrategia, tanto si está destinada a la formación como a la inserción laboral, depende de su ajuste a la problemática y condiciones específicas de la población a la que va destinada. De ahí la necesidad de establecer pautas o recomendaciones obtenidas a partir de la experiencia y los resultados de la investigación con objeto de mejorar la interacción social de estas personas y facilitar la comunicación en los ámbitos y programas en los que trabajamos con estos colectivos.

Como no es nuestra pretensión recoger exhaustivamente todas las sugerencias que suelen hacerse, nos vamos a referir esencialmente a dos de los trabajos donde se plantea con claridad este tema. En el primero de ellos, Paton (1996) destaca la importancia de desarrollar estrategias de compensación ante las dificultades auditivas y propone una serie de recomendaciones:

1. facilitar su ubicación lejos de los ordenadores o en zonas donde haya una buena recepción acústica;

2. aconsejar la utilización de los instrumentos tecnológicos que les puedan ser útiles, como el uso del audífono, y contar con alguien que los apoye en los trabajos que implican lecturas;

3. tocarles en el hombro antes de iniciar una conversación;

4. ajustar el volumen de teléfonos y de alarmas;

5. proporcionar la información por escrito;

6. sugerirles tomar notas y apuntar en una agenda lo que deben hacer a largo plazo;

16. Por ejemplo, los beneficios de las adaptaciones protésicas están condicionados al hecho de que se prescriban, se puedan adquirir, y de su aceptación y uso por parte de las personas con deficiencias auditivas. 
7. enseñarles las claves para aprender a interpretar los sentimientos de la gente y cómo cambiar el lenguaje respecto a ellos.

El segundo que queremos destacar es el realizado en nuestro contexto por Flórez y otros (1998) que también proporciona una serie de recomendaciones o pautas que deben ser tenidas en cuenta por todos aquellos que trabajen como formadores o en el empleo con personas con deficiencias auditivas (empresarios y otros compañeros de trabajo). Subrayan que en la medida en la que el formador pueda ir controlando todos estos aspectos, el nivel de aprendizaje y el rendimiento laboral de la persona con deficiencia auditiva mejorará sensiblemente.

Tomando como referencia ambos estudios y las observaciones obtenidas de la bibliografía comentada, vamos a concluir este apartado efectuado una serie de propuestas que el equipo de investigación considera oportunas a la vista de los datos recabados a través de la investigación realizada y de su experiencia profesional en este campo. Las propuestas se formulan a modo de recomendaciones o pautas que pueden ser muy útiles para trabajar con personas con deficiencias auditivas. De modo especial queremos destacar las siguientes:

\section{Cuidar el ambiente o medio donde se produce la comunicación:}

- No olvidar que el sordo recibe la información visualmente: por lectura labial, información escrita, expresiones corporales y faciales, etc.

- Situar a la persona en el lugar en el que mejor pueda percibir, a través de sus restos auditivos, favoreciendo la lectura labial y el acceso visual a la información. Combinar las posiciones «cerca del formador», «frente al formador»y «visión global de la clase y sus compañeros».

- Es conveniente que exista una buena iluminación y evitar los reflejos para facilitar la lectura labial.

- Evitar las vibraciones y las áreas ruidosas para que estas no interfieran en el aprovechamiento de los restos auditivos.

\section{Atender a los aspectos organizativos de la comunicación:}

- El formador debe procurar estar frente al alumno o trabajador para permitir una buena lectura labial. Es importante en todo momento controlar su orientación, debiendo evitar pasear, girarse mientras habla, hablar de espaldas al sujeto, etc.

- Evitar las posturas y aquellos elementos que impidan ver directamente la cara de las personas que están hablando: barbas y bigotes demasiado largos, interferencia de manos, papeles, bolígrafos, cigarros, etc., que puedan suponer un obstáculo.

- Procurar el contacto visual antes de comenzar la comunicación.

- Señalar las fuentes de información o la persona que está hablando.

- Tratar de articular de forma clara y a una velocidad moderada, no exagerando ni la forma de pronunciación habitual ni los movimientos.

- Emplear un lenguaje lo más natural posible y adecuado siempre al interlocutor.

- Evitar corregir continuamente sus errores esperando a que termine de hablar.

- Aunque la persona con deficiencia auditiva lleve una prótesis auditiva no siempre controla su voz, siendo necesario darle información sobre cuando debe subir o bajar la intensidad. 
- Utilizar movimiento corporal, gestos o mimo para llamar la atención del alumno y facilitar la comprensión.

- Emplear ilustraciones y diagramas siempre que sea posible, tanto en la comunicación como en la organización del espacio.

- Escribir palabras clave para facilitar su comprensión y correcta asimilación.

- Procurar obtener constantemente información, «feed back» del alumno acerca de su comprensión.

- Cambiar las palabras cuando se repita una información que no se haya entendido.

\section{Mejorar las relaciones sociales:}

- Realizar siempre que sea posible el trabajo en pequeños grupos para favorecer la relación y participación con sus compañeros. Buscar la relación con quienes pueda encontrar mayor facilidad comunicativa, tanto por su empatía como por la recepción de una lectura labial clara.

- Darles la oportunidad de participar en las clases o sesiones de formación, acostumbrándose a sus diferentes formas de hablar, teniendo en cuenta que aunque al principio se tarda algún tiempo, puede ser muy beneficioso a medio y largo plazo.

- Proporcionar a los compañeros de clase y de trabajo información sencilla y clara sobre las personas con deficiencias auditivas y sobre sus necesidades.

- Evitar la sobreprotección. Hay que facilitar que los compañeros ayuden a la persona sorda pero sólo en aquellos aspectos que sabemos que puede tener dificultades.

- Favorecer y estimular el proceso de aceptación de la deficiencia auditiva, siendo necesario para ello mostrar nuestra confianza en sus posibilidades.

\section{Facilitar dinámicas de trabajo positivas:}

- En caso de tener que tomar apuntes, se recomienda situar al alumno con deficiencia auditiva junto a un compañero oyente para que pueda ayudarle.

- Debe tenerse en cuenta que no puede escuchar y escribir o ejecutar otra tarea al mismo tiempo.

- Controlar el ritmo de debate de manera que «la persona sorda» no se sienta perdida ante un ritmo acelerado. Utilizar técnicas para retomar y repetir los puntos de discusión.

- Dejarle períodos de descanso para evitar la fatiga visual. No olvidar que mantiene una atención constante para realizar la lectura labial.

- Se le pueden aclarar sus dudas mientras sus compañeros realizan alguna tarea, no posponiéndolas para luego no aclararlas.

- Es importante señalar los cambios de actividad o de tema de forma muy clara.

- Se le debe informar sobre las normas y las reglas del trabajo, así como de las posibles modificaciones, comprobando que ha comprendido lo que se le ha dicho. Es conveniente que disponga de esta información por escrito. 
- Los métodos activos son los que propician su máxima participación, debiendo favorecerse la enseñanza basada en la observación y en la práctica.

\section{Reforzar los contenidos básicos:}

- Es fundamental planificar el contenido de lo que se va a exponer, como por ejemplo, el vocabulario introducido por primera vez, el lenguaje técnico, etc. Es muy recomendable presentar los contenidos teóricos que se consideran básicos e imprescindibles de forma escrita.

- Es muy importante saber que en ocasiones la repetición de lo dicho o explicado no mejora la comprensión, siendo lo más adecuado expresar el mensaje con otras palabras.

- Utilizar esquemas (partiendo de las ideas generales a las particulares) y destacar las ideas importantes de los textos en negrita.

- Evaluar a los alumnos con deficiencias auditivas sobre las mismas bases que a los oyentes exigiendo las mismas responsabilidades.

\section{Preparar materiales de apoyo apropiados:}

- Son de gran utilidad las ayudas visuales, como el uso de la pizarra, documentos escritos, gráficos, retroproyector, etc. Estos materiales resultan especialmente interesantes cuando se habla de algo nuevo.

- En la organización espacial es importante el uso de paneles informativos gráficos.

- Hacer frecuente el manejo del diccionario para facilitar la comprensión y ampliar el vocabulario.

\section{Adaptar los textos escritos:}

- Adaptar los textos cuando sea necesario:

- Seccionar las oraciones demasiado largas.

- Reducir la dificultad del vocabulario.

- Cuando se utilizan pronombres u otros términos que se refieran a una palabra usada anteriormente, asegurarse de que el referente está claro.

- Utilizar conjugaciones simples. Cuando no haya otro remedio que utilizar una palabra difícil, poner entre paréntesis su significado o un sinónimo (el criterio de frecuencia puede ser muy útil).

- Concretar las preguntas sin que sean demasiado largas.

- Colocar gráficos, dibujos, etc. cerca del texto al que se refieren.

- Asegurarse de que la idea y contexto general que se va a tratar está claro, pudiendo ayudarnos con esquemas y con diferentes colores que separen la idea principal de las secundarias.

- Las técnicas mnemotécnicas pueden resultar muy útiles.

\section{Utilizar las ayudas técnicas disponibles:}

- En algunos casos son necesarias adaptaciones en el puesto de trabajo o en aula para ayudar a las personas con deficiencias auditivas a rendir de forma adecuada y evitar riesgos innecesarios y previsibles. Algunas de estas adaptaciones son: 
- Señalización clara y abundante en su ambiente laboral.

- Sistemas de emergencia luminosos.

- Sistemas de avisos mediante rótulos o paneles cuando en el edificio se utilice un sistema de megafonía para comunicarse con los empleados.

- Teléfono de texto o fax.

- Sistema de señalización y alarmas vibrotáctiles.

\section{Referencias bibliográficas}

Aguado, A. L. (1995): Historia de las deficiencias. Madrid, Escuela Libre Editorial.

Alvira Martín, F., Cruz Chust, A., Blanco Moreno, F. (2000): Los problemas, necesidades y demandas de la población con discapacidad auditiva en España: una aproximación cualitativa. Madrid, Ministerio de Trabajo y Asuntos Sociales, Observatorio de la Discapacidad.

Anderson, G. B., McGEE, S. (1998): Creating School to Work Initiatives for Deaf Students, Perspectives in Education and Deafness, 16 (5), 4-7.

Allen, T. E. (1992): Subgroup Differences in Educational Placement for Deaf and Hard of Hearing Students, American Annals of the Deaf, 137 (5), 381-388.

Backenroth, G. A. M. (1995): Deaf People's Perception of Social Interaction in Working Life, International Journal of Rehabilitation Research, 18 (1), 76-81.

Backenroth, G. A. M. (1997): Deaf employess' Empowerment in Two Different Communication Environments, International Journal of Rehabilitation Research, 20 (4), 417-419.

Bullis, M., Reiman, J. (1992): Development and Preliminary Psychometric Properties of the Transition Competence Battery for Deaf Adolescents and Young Adults, Exceptional Children, 59 (1), $12-26$.

Busby, H. R., Danek, M. M. (1998): Transition: Principles, Policy, and Premises, Perspectives, Education and Deafness, 16 (5), 8-11.

Cambra, C.: A Comparative Study of Personality Descriptors Attributed to the Deaf, the Blind and Individuals with no Sensory Disability, American Annals of the Deaf, 141 (1) (1996), 24-28.

Cohen, O. P., Ed. (1995): The Adverse Implications of Full Inclusion for Deaf Students. New-York. International Congress on Education of the Deaf.

Comision Europea (1996): Helios II. Guía Europea de Buena Práctica. Hacia la igualdad de oportunidades de las personas discapacitadas. Bruselas, Oficina de Publicaciones de las Comunidades Europeas, 1996.

Congress of the U.S.(1992): Field Hearing on Rehabilitation. Services and Education of the Deaf Programs. Washington, House Committee on Education and Labor.

Dabbs, C. (1991): Meeting Multiple Challenges: To Work, To Study, and To Succeed, Perspectives in Education and Deafness, 10 (1), 10-11.

De Miguel, M., Pereira, M., Portilla, A., Rivas, A., Valdivieso, M. J. (2000): Necesidades y problemas de la población con deficiencias auditivas en el proceso de integración en el mundo laboral.La realidad en Asturias. Oviedo, Servicio de Publicaciones de la Universidad de Oviedo.

DeGraw, C. E., Stroud, L. H (1993): The Macintosh Computer: A Link between Work and the Disabled Worker. Virginia, American Vocational Association Convention. 
Downie, M., Moore, P. (1998): Closing the Gap: Schools Forge a Bridge to Community- In California, Comprehensive Services and Realistic Planning. Perspectives in Education and Deafness, 16 (5), 15-16.

El-Khiami, A. (1993): Employment transition and Establishing Careers by Postsecondary Alumni with Hearing Loss, Volta Review, 95 (4), 357-366.

Flórez García, M. A., González González, I., Santos González, A. M., Alcedo Rodríguez, M. A., Aguado Díaz, A. L. (1998): Las personas con deficiencia auditiva, El formador ante la discapacidad. Manual para profesionales. Avilés, Fondo-Formación Asturias, Principado de Asturias.

Gateway Technical Institute (1993): Access: Special Instructional Support Services. A Faculty Handbook. Wisconsin, Gateway Technical Inst.

Galvin, J. C., Scherer, M. J. (1996): Evaluating, Selecting and Using Appropriate Assistive Technology. Maryland, Aspen Publishers.

Gilliam, J., Easterbrooks, S. (1997): Educating Children Who Are Deaf or Hard of Hearing: Residential Life, ASL, and Deaf Culture. Washington, Office of Educational Research and Improvement.

Gregory, S.(1992): The Language and Culture of Deaf People: Implications for Education, Language and Education, 6 (2-4), 183-97.

Grupo de investigación y apoyo a la educación de las personas sordas (1998): Situación educativa de las personas sordas en Aragón. Zaragoza, CPR de La Almunia de Doña Godina.

Hefu, R. Getty, L (1993): Overcoming Difficulties Experienced in the Work Place by Employees with Occupational Hearing Loss, Volta Review, 95 (4), 391-402.

Herrés, H. (1982): Estudio sobre la Educación de Niños y Adolescentes Sordos. Barcelona, Médica y Técnica.

Hintermair, M. (2000): Children Who Are Hearing Impaired with Additional Disabilities and Related Aspects of Parental Stress, Exceptional Children, 66 (3), 327-332.

Hites, J. (1994): On Being Hearing Impaired in the Work place, Performance and Instruction, 33 (5), $10-12$.

Hutchins, P. (1996) Rosie's Walk: Sign Language CD-ROM. Texas, Texas School for Deaf.

IMSERSO (1999): Glosario de Términos. http://www.mtas.99.

INSERSO (1996): Plan de Acción para las Personas con Discapacidad (1997-2002). Madrid, Ministerio de Trabajo y Asuntos Sociales.

Instituto Nacional de Estadística (1987): Encuesta sobre deficiencias, discapacidades y minusvalias. Madrid, I.N.E., 1987.

Jaitly, M., Sims, N. (1998): Closing the Gap: Schools Forge a Bridge to Community- In Colorado: Pathership With Community College Enables Students' Successful Transitions, Perspectives in Education and Deafness, 16 (5), 14.

Klee y otros (1994): A Kentucky Response to the ADA, Training and Development, 48 (4), 48-49.

Kolvitz, M., Ed. (1996): Focusing on Staff Development and Administrative Issues. Tennessee. Biennial Conference on Postsecundary Education for Persons Who Are Deaf or Hard of Hearing.

Kolvitz, M., Ed. (1996): Focusing on Non-Traditional and Underserved Students. Tennessee. Biennial Conference on Postsecundary Education for Persons Who Are Deaf or Hard of Hearing.

Kolvitz, M., Ed. (1996): Developing Career Planning and Placement Strategies. Tennessee. Biennial Conference on Postsecundary Education for Persons Who Are Deaf or Hard of Hearing.

Lam, K. H. y otros (1994): Young Deaf Adults and the Transition from High School to Postsecondary Careers. Washington D.C., Gallaudet Research Institute Occasional. 
Long, G., Clark, D. A. (1993): Defining Traditionally Underserved Persons who are Deaf. Washington, National Institute on Disability and Rehabilitation Research.

Mackie, A. (1993): Manual visual Communication in Adult Education: A Case Study Analysis, Australian Journal of Adult and Community Education, 33 (2), 122-132.

Mason, D. G. (1994): Bilingual/Bicultural Deaf Education Is Appropriate. Occasional Monograph Series, Number 2. Toronto (Ontario), York University.

Mason, D. G. (1997): Mainstream Education And Deaf Students, CAEDHH-Journal La Revue ACESM, 23 (2-3), 95-118.

McBain, S., Phillips Jones, L. (1985): Enhance Understanding of Individuals with Disabilities. Columbus, The National Center for Research in Vocational Education.

McLeod, G., Gallinger, J. E. (1992): The Career Status of Deaf Women: A Comparative Look, American Annals of the Deaf, 137 (4), 315-325.

Ministerio de Trabajo y Asuntos Sociales (2000): Guía Laboral y de Asuntos Sociales 2000. Madrid, MTAS.

Minnett, A. y otros (1994): Play Behavior and Communication between Deaf and Hard of Hearing Children and their Hearing Peers in an Integrated Preschool, American Annals of the Deaf, 139 (4), 420-429.

Minnesota University (1997): Project Forward. School to Work Outreach Project 1997 Exemplary Model/Practice/Strategy. Minnesota, Minneapolis Institute on Community Integration.

Minnesota University (1998): Career Help at Overbrook for Individuals who Choose Employment. School to Work Outreach Proyect 1998 Exemplary. Minnesota, Minneapolis Institute on Community Integration.

Mowry, R. L., Anderson, G. B. (1993): Deaf Adults Tell Their Stories: Perspectives on Barriers to Job Advancement and On-the-Job Accommodations, Volta Review, 95 (4), 367-377.

Musselman, C. y otros (1996): The social Adjustment of Deaf Adolescents in Segregated, Partially Integrated and Mainstreamed Settings, Journal of Deaf Studies and Deaf Education, 1 (1), 52-63.

Nathanson, J. H. (1991): Postsecondary Education for Individuals with Disabilities, OSERS-News, 4 (1).

NCDA(1993): Career Development: A Policy Statement of the 1992-1993 Board of Direction. Alexandria, VA, National Career Development Association.

Nowell, R., Innes, J. (1997): Educating Children Who Are Deaf or Hard of Hearing: Inclusion. Eric Digest \# 557. Washington, Office of Educational Research and Improvement.

Observatorio de la discapacidad (1999): Empleo y Discapacidad, MINUSVAL, 11-18.

Olson, J. R. (1992): The Garden Cafe: An Experiment in Vocational Education in the Philippines, American Annals of the Deaf, 137 (3), 283-287.

Organización Mundial de la Salud (1994): Clasificación internacional de deficiencias, discapacidades y minusvalías. Manual de clasificación de las consecuencias de la enfermedad. Madrid, INSERSO. ( $2^{\mathrm{a}}$ edición).

Paton, J. H. (1996): Living and Working with a Central Auditory Processing Disorder (CAPD). California, http://www.Ldonline.org.

Pereda, C., De Prada, M. A., Actis, W., Santamarina, C., Camas, V. (1998): Discapacidad y trabajo en España. Estudio de los procesos de inclusión y exclusión social de las personas con discapacidad. Madrid, Ministerio de Trabajo y Asuntos Sociales.

Reiman, J. y otros (1992): Assessment, Intervention, and Program Needs of Lower Achieving and Multiply Disabled Deaf People Requiring Extended Transition Support. Illinois, Delkalb. 
Resa, S. (1999): Las personas sordas, por la educación bilingüe, Minusval, (118), 64-65.

Ryall, G. (1993): Literacy for Deaf People Seeking Employment, TESL-Talk, 21 (1), 48-55.

Schildroth, A. y otros (1991): Deaf students In Transition: Education and Employment Issues for deaf Adolescents, Volta Review, 93 (5), 41-53.

Schoroedel, J. (1992): Helping Adolescents and Young Adults Who Are Deaf Make Career Decisions, Volta Review, 94 (1), 37-46.

Schulte, E., Ed. (1995): Technical Education for Deaf Students around the World at College/University Level: The German Example. German, Annual Meeting of the International Congress on Education.

Spencer, P. y otros (1994): Communicative Interactions of Deaf and Hearing Children in a Day Care Center: An Exploratory Study, American Annals of the Deaf, 139 (5), 512-518.

Stewart, D. A. y otros (1991): Using Instructional Design Principles to Procedure an IVD to Enhance the Linguistic Environment of Deaf Children, Journal of Special Education Technology, 11 (3), 121-135.

Stewart, D. A. y otros (1996): Support for Language and Communication, Perspectives in Education and Deafness, 14 (3), 11-17.

Takala, M. (1995): The Say I'm Stupid, but I Just Don't Hear: Hearing Impaired Adults view of Finish Society. Research Report 142. Finland, Helsinki University.

Texas Higher Education Coordinating Board (1994): Realtime Training: Refinement/Implementation/Marketing Final Report. Austin, Texas. Texas Higher Education Coordinating Board.

Thompson, A. R., Bethea, L. L., Rizer, H. F. (1998): Students with Disabilities and Assistive Technology: A Desk Reference Guide. Hutto, M.D. Mississippi, Mississippi State Departament of Rehabilitation Services.

Tucker, B. (1993): Deafness:1993-2013 The Dilema, Volta-Review, 95 (2), 105-108.

Vialle, W., Paterson, J. (1996): Constructing a Culturally Sensitive Education for Gifted Deaf Students. Australia, Conference of the Australian for the Education of the Gifted and Talented.

Warick, R. (1994): Jones Memorial Lecture in Deafness between Two Worlds: A Hard of Hearing Perspective, ACEHI-Journal Revue ACEDA, 20 (1-2), 49-57.

Woodrick, W. E., Wolfe, V. L., Ed. (1995): The PEC (Postsecondary Education Consortium) Salutes Success. Tennessee, Postsecondary Education Consortium.

Zahn, S. B., Kelly, L. (1995): Changing Attitudes about Employability of the Deaf and Hard of Hearing, American Annals of the Deaf, 140 (5), 381-385. 